\title{
Stereoselective Synthesis of Diverse $\alpha$-Hydroxy- $\beta$-amino Acids and It's Application for Synthesis of Dipeptide Expecting as a Protease Inhibitor
}

\author{
Sang Hun Jang, Ju Young Kim, Min Kyu Kim, Jeong Woo Han, Ki Hun Park, ${ }^{+}$Yong-Jin Yoon,;** \\ and Sang-Gỵeong Lee ${ }^{*}$
}

Department of Chemistry and Research Institute of Life Science, Graduate School for Molecular Materials and Nanochemistrv, Gieongsang National Universitu, Jinju 660-701. Korea. 'E-mail: leesangàgnu. ac.kr - Department of Agricultural Chemistry and Division of Applied Life Science. Gveongsang National Chiversitu, Jiniu 660-701, Korea

$\ddagger$ Department of Chemistry and Research Institute of Katural Science, Graduate School for Molecular Materials and Nanochemistry, Gveongsang National Chiversin, Jinju 660-701, Korea

Received September 4. 2008. Accepted November 18. 2008

\begin{abstract}
Few $\alpha$-hydroxy- $\beta$-amino acids were synthesized via various nucleophilic addition of the epoxide and followed by stereoselective nucleophilic substitution reaction and eliminative cleavage of the acetal selectively in diacetal compound. One of the synthesized $\alpha$-hydroxy- $\beta$-amino acid reacted with L-leucine methylester to give corresponding dipeptide in good yields.
\end{abstract}

Key Words: $\alpha$-Hydroxy- $\beta$-amino acids, Nucleophilic addition. Epoxide ring opening. Eliminative cleavage

\section{Introduction}

Amino alcohols especially vicinal $s m$ - and anti-hỵdroxy amino units are an important class of compounds. Because of these units are constituents of many natural products such as paclitaxel (taxol). KRI 1230 and KRI 1314. In recent years, $\$ 1 n-\alpha$-hydroxy- $\beta$-amino acids like cyclohexylnorstatine 1 have received considerable interest from a pharnaceutical point of view. ${ }^{l}$ Because of cyclohexylnorstatine is the C-terminal residue of KRI $131+2$. a tripeptide with potent rennin inhibitory activity (Fig. 1) ${ }^{2,3}$

Many methods have already been developed for the synthesis of these $\alpha$-hydroxy- $\beta$-amino acid units. ${ }^{\top}$ Most of the early studies used chiral natural products as starting materials were limited in their flexibility of stnictural modification. In

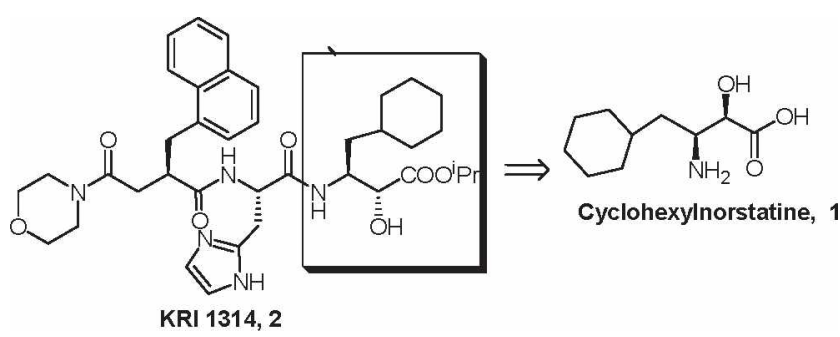

Figure 1. Cyclohexylnorstatine 1 is one of the part of the KRI 1314 which is potent reninu indibitor.

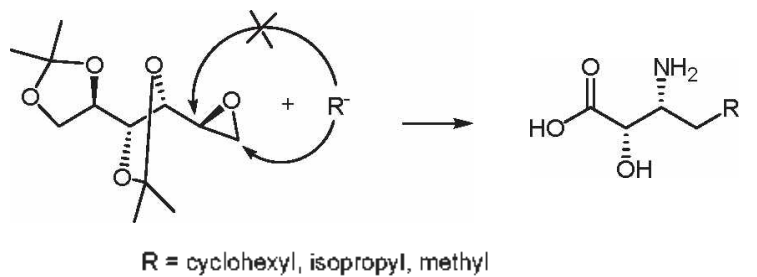

Figure 2. Regioselective epoxide ring opening reaction for synthesis of diverse $\alpha$-hudroxt- $\beta$-amino acids. connection with these points. we noticed that a nucleophilic addition of epoxide is very useful methodology for synthesis of various $\alpha$-lyydrosy- $\beta$-anuino acids.

In this paper. we wish to report synthetic routes for $\alpha$-hy'droxy- $\beta$-amino acid derivatives via the various nucleophilic addition of epoxide and followed by regio- and stereoselective nucleophilic substitution reaction and elininative cleavage of the acetal selectively in diacetal compounds (Fig. 2).

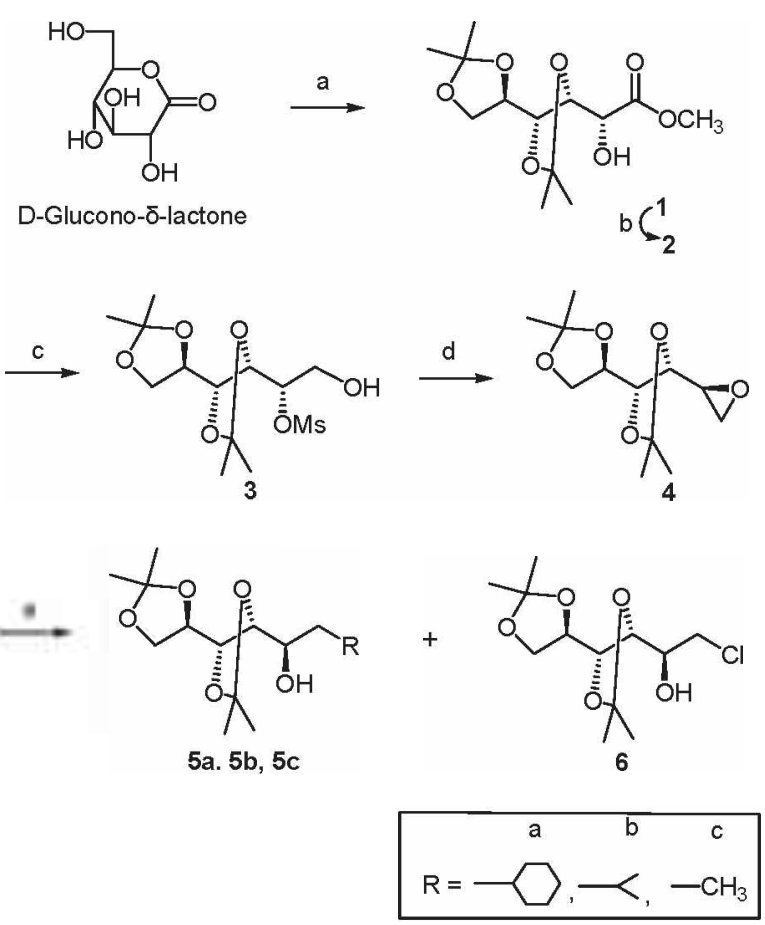

Scheme 1. Reagents and conditions; a) 2,2-Dimethoxypropane, $\mathrm{MeOH}$, Acetone, TsOH, $\mathrm{H}_{2} \mathrm{O}, \mathrm{rt}, 97 \%$, b) MsCl, Et $\mathrm{N}, \mathrm{CH}_{2} \mathrm{Cl}_{2}, 0^{\circ} \mathrm{C}$, rt, $98 \%, \mathrm{c}) \mathrm{NaBH}_{4}, \mathrm{THF}, 0^{\circ} \mathrm{C}, 95 \%$ d) $\mathrm{NaOMe}, \mathrm{MeOH}, \mathrm{rt}, 87 \%$. e) $\mathrm{RMgX}, \mathrm{CuI}, \mathrm{THF},-10^{\circ} \mathrm{C}$ to rt, $80 \%$. 


\section{Results and Discussion}

The compound 1 prepared easily by the usual method from $D$-glucono-ò-lactone. After mesylation and ester reduction of 1. treated with $\mathrm{NaOMe}$ to afford epoxide + in quantitative yield (Scheme 1).

To open epoxide ring of 4 , we reacted various nucleophiles such as alkyl lithiums. Grignard reagents and Gilman reagents. Treatment of epoxide 4 with alkyl lithium reagents (methỵl lithium. isopropyl lithium and cyclohexyl lithium) gave many undesired product as mixture of stereoselectively and regioselectively uncontroled product. On the other hand. treatment of $\mathbf{4}$ with Grignard reagents (methỵl, isopropyl and cyclohexyl Grignard reagents) gave unexpected the halogen attacked compound $(6 . \mathrm{X}=\mathrm{Cl}$ ) as major product instead of desired 5a-c. After several unsuccessful trials, the ring opening reaction of epoxide + using Grignard reagents in presence of copper(I) iodide gave alcohol $\mathbf{5}$ as major product (Table 1). According to these results, we thought the copper(I) iodide is useful for the regioselective ring opening of 4 . Under the these consideration, we reacted 4 with cuprates (Gilman reagent) to give desired product $\mathbf{5 a - c}$ in reasonable yield.

The alcohol 5 was converted to azido compound $\mathbf{8}$ through mesylation and $\mathrm{S}_{\mathrm{N}} 2$ intramolecular displacement by sodium azide. The azido compound 8 was hydrogenated with $\mathrm{H}_{2}$ in the presence of $10 \% \mathrm{Pd} / \mathrm{C}$ followed by protection of amino group with 9-phenyl-9-fluorenyl (Pf) bromide to give $\mathbf{1 0}$ in good yield (Scheme 2) ${ }^{8}$ At this stage, we introduced the Pf group as a protection of amino group because there are remains many steps should be proceed under the strong acid conditions.

The selective cleavage of terminal isopropylidene group of $\mathbf{1 0}$ was proceed in $70 \%$ acetic acid condition to give $\mathbf{1 1}$ in good yield. ${ }^{9}$ The diol 11 was converted to alcohol 12 by using $\mathrm{NaIO}_{4}$ and $\mathrm{NaBH}_{4}$. The alcohol 12 was mesylated and followed by substitution with lithium iodide to give 14. The isopropy lidene iodide $\mathbf{1 4}$ was treated with $n$-BuLi to give ally lic alcohol 15 through simultaneous elimination reaction. Ally lic alcohol 15 was protected with benzyl bromide and followed by dihydroxylation of alkene with $\mathrm{OsO}_{4}$ to give corresponding diol. ${ }^{1,9}$ The obtained diol compound oxidizied with $\mathrm{NaIO}_{4}$ to give aldehyde and followed by oxidation with $\mathrm{KMnO}_{4}$ resulted to desired $\alpha$-hydroxy- $\beta$-anuno carboxylic acid $\mathbf{1 7}$ in good yield. The 17a has different stereochemistry with cyclohexyinorstatine (1) but it has the same skeleton and functional groups and $\mathbf{1 7 b}$ has the same skeleton and functional groups with $(2 S$. $3 R$ )-3-amino-2-hydroxy-5-methylhexanoic acid which is the $N$-terninal amino acid of amastatin ${ }^{11}$ that a tripeptide which has been found to inhibit leucine aminopeptidase and aminopeptidase $\mathrm{A}^{\text {]? }}$

Among of $17 \mathrm{a}, 17 \mathrm{~b}$ and $17 \mathrm{c}$. compound 17a reacted with L-leucine methylester under the presence of DCC. HOBT and $\mathrm{Et}_{3} \mathrm{~N}$ in THF to give desired dipeptide 18 in good yield. $\mathrm{Hy}$ 'droly sis of 18 by LiOH in mixed solvent (THF : $\mathrm{H}_{2} \mathrm{O}=2$

Table 1. Ring Opening Reaction of Epoxide 4 by Various Nucleophiles.

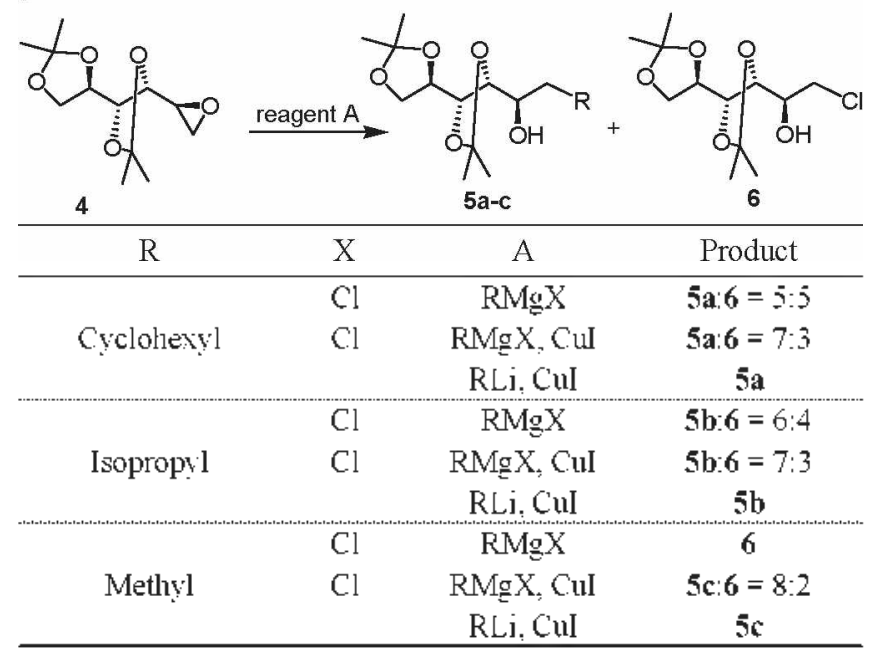

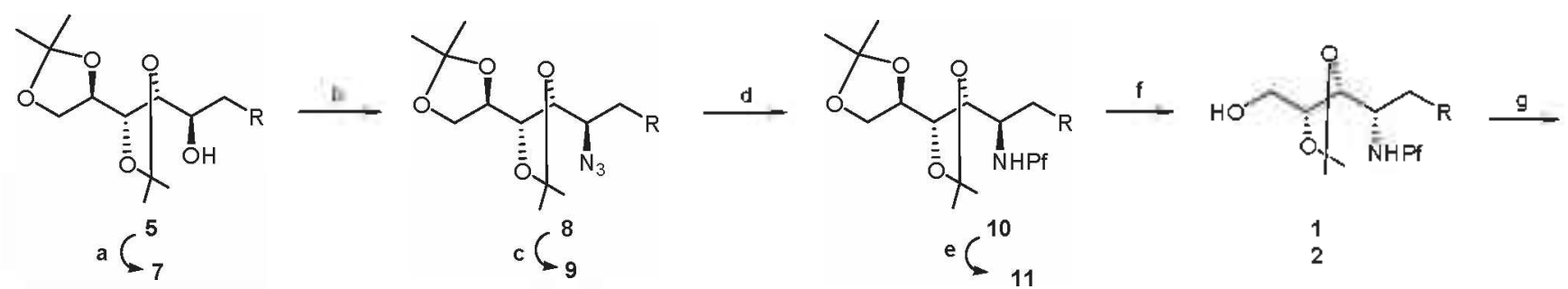

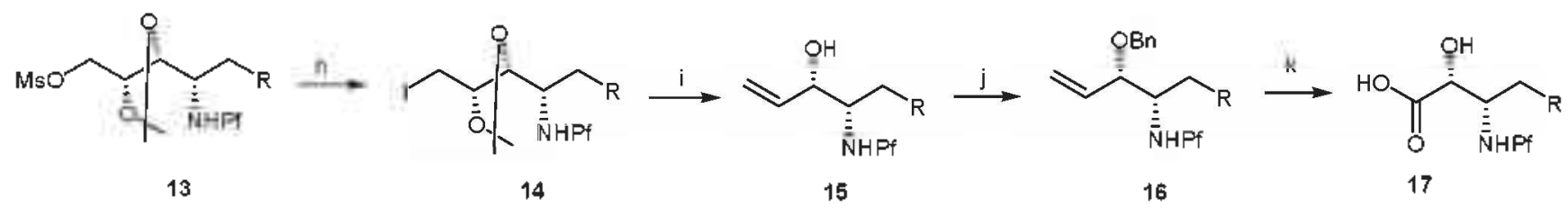

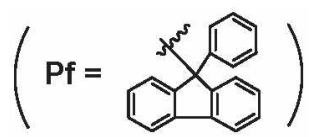

Scheme 2. Reagents and conditions; a) Ms-Cl, Et $3 \mathrm{~N}_{2} \mathrm{CH}_{2} \mathrm{Cl}_{2}, 0^{\circ} \mathrm{C}, 98 \%$, bj $\mathrm{NaN}_{3} \mathrm{DMF}, 80^{\circ} \mathrm{C}, 87 \%$ c) $10 \% \mathrm{Pd} / \mathrm{C}, \mathrm{H}_{2}, \mathrm{MeOH}_{\text {, it }} 95 \%$, dj $\mathrm{Pt}-\mathrm{Br}$,

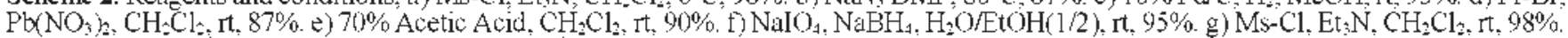

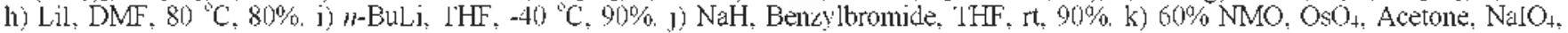
$\mathrm{EtOH} / \mathrm{H}_{2} \mathrm{O}(1 / 1), \mathrm{K}_{2} \mathrm{CO}_{3}, \mathrm{KMnO}_{4}, \mathrm{THF} / \mathrm{H}_{2} \mathrm{O}(1 / 1), 1 \mathrm{t}, 75 \%$ 
<smiles>N[C@@H](CC1CCCCC1)[C@H](O)C(=O)O</smiles>

$17 a$<smiles>COC(=O)[C@H](CC(C)C)NC(=O)[C@H](OCc1ccccc1)[C@H](N)CC1CCCCC1</smiles>

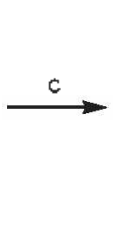

20

Scheme 3. Reagents and conditions; a) TsOH, L-len-OCHs, HOBT, DCC, Ets N, THF, $0^{\circ} \mathrm{C}, 75 \%$. b) LiOH, THF/H $\mathrm{H}_{2} \mathrm{O}(2 / 1)$, $0{ }^{\circ} \mathrm{C}$. c) $10 \%$ $\mathrm{Pd} / \mathrm{C}, \mathrm{H}_{2}, \mathrm{MeOH}, 70-80^{\circ} \mathrm{C}, 83 \%$.

1) to give acid 19 in quantitative yield. The obtained 19 was deprotected with $\mathrm{H}_{2}-\mathrm{Pd} / \mathrm{C}$ in $\mathrm{MeOH}$ at $70-80^{\circ} \mathrm{C}$ to give desired peptide 20 with $83 \%$ yield (Scheme 3 ).

In conclusion we reported the result of regioselective reaction of epoxide ring opening by nucleophiles attack. According to survey. regioselective ring opening of epoxide was completed more easily under the conditions of Gilman reagents than Grignard reagents. And also, we synthesized dipeptide $\mathbf{2 0}$ which expecting as a protease inhibitor

\section{Experiments}

Genenal. All solvents were purified before use with standard drying procedures, unless otherwise specified. Reactions were monitored by TLC using Merck silica gel 60 F-254 plates with UV indicator or/and visualized with phosphomolybdic acid $(10 \%$ solution in EtOH). All the organic layers were dried over $\mathrm{Na}_{2} \mathrm{SO}_{4}$ before concentration in vacuo. Column chromatography was carried out using $230-400$ mesh silica gel. Elemental analy sis or $\mathrm{C}$. $\mathrm{H}$ and $\mathrm{N}$ are in agreement with the theoretical data. except for compounds containing halogens. where combustion analysis could not be performed. Melting points were measured on Thomas-Hoover melting point apparatus but the temperatures were not corrected. ${ }^{1} \mathrm{H}$ - and ${ }^{13} \mathrm{C}-\mathrm{NMR}$ experiments were conducted on Bruker AW-500 spectrometer. Optical rotation were measured on a Jasco DIP- 1000 polarimeter and $[\alpha]_{D}$ values are given in units of $10^{-1} \mathrm{degcm}^{2} \mathrm{~g}^{-1}$.

Preparation of methyl 3,4;5,6-di- $O$-isopropylidene-2- $O$-methanesulfonyl- $D$-gluconate $(1)$ and methyl 3,$4 ; 5,6$-di- $O$-isopropylidene-2- $O$-methanesulfonyl- $D$-gluconate (2) were prepared according to ref. ${ }^{13}$ and physical and spectral dates were correlated well with previously reported

Methyl 3,4;5,6-di- $O$-isopropylidene-2- $O$-methanesulfonylD-glucitol (3). To a solution of compound 2 (2.22 g. 6.03 mmol) in $\mathrm{MeOH}(30 \mathrm{~mL})$ was added portionwise $\mathrm{NaBH}_{4}(0.68$ g. $18.1 \mathrm{mmol}$ ) at $0^{\circ} \mathrm{C}$ for $3 \mathrm{~min}$ and stimed for $11 \mathrm{~h}$ and then quenched with water $(5 \mathrm{~mL})$. The reaction mixture was extracted with EtOAc $(3 \times 30 \mathrm{~mL})$ and the organic layers were evaporated in vacuo. The combined organic extracts were dried with $\mathrm{Na}_{2} \mathrm{SO}_{4}$ and evaporated under vacuo. The crude mixture was purified by flash column chromatography (hexane/EtOAc, 9: 1. . $/ \mathrm{v})$ to afford pure $3(2.03 \mathrm{~g} .98 \%$ ) as an oil. $[\alpha]_{D}=-6.3\left(c 2.20, \mathrm{CHCl}_{3}\right):$ IR (KRS-5) 3490, 2980, 2970. $2900,1370,1360,1300,1290,1280,1100,990 \mathrm{~cm}^{-1}:{ }^{1} \mathrm{H} N \mathrm{NR}$ $\left(500 \mathrm{MHz} \mathrm{CDCl}_{3}\right)$ ò $1.35(\mathrm{~s}, 3 \mathrm{H}), 1.39$ (s. 3H). $1.41(\mathrm{~s}, 3 \mathrm{H})$. 1.44 (s. 3H). 2.64-2.67 (t. $-\mathrm{OH}, J=6.7 \mathrm{~Hz}) .3 .14$ (s. $3 \mathrm{H})$. 3.92-3.97 (m, $4 \mathrm{H}), 4.04-4.07(\mathrm{~m} .1 \mathrm{H}) .4 .18-4.24(\mathrm{~m}, 2 \mathrm{H}), 4.82$ (q. $1 \mathrm{H}, J=4.4 \mathrm{~Hz}):{ }^{13} \mathrm{C} \mathrm{NMR}\left(125 \mathrm{MHz}, \mathrm{CDCl}_{3}\right) \hat{o} \mathrm{l} 10.6$. $110.3 .81 .9 .79 .9 .77 .8 .76 .8 .68 .1,63.0 .38 .9 .30 .9 .27 .2 .26 .6$.
26.3. 25.2. Anal. Calcd for $\mathrm{C}_{13} \mathrm{H}_{2} \mathrm{O}_{8} \mathrm{~S}_{3}: \mathrm{C}, 45.87 ; \mathrm{H}, 7.11$ Found : C. 45. 86 H, 7.10.

1,2-Anhy dro-1,2-epoxy-3,4;5,6-O-diisopropylidene$D$-glucose (4). To a stirred solution of compound $3(4.4 \mathrm{~g} .12 .9$ nmol) in $\mathrm{MeOH}(50 \mathrm{~mL})$ was added portionwise Na metal $(0.45 \mathrm{~g} .19 .4 \mathrm{mmol})$ at $\left({ }^{\circ} \mathrm{C}\right.$. After stirring $2 \mathrm{lh}$ at rt., water $(10$ $\mathrm{mL})$ was slowly added and extracted with EtOAc $(3 \times 40 \mathrm{~mL})$. The combined organic extracts were washed with water and brine and dried over anhydrous $\mathrm{MgSO}_{4}$ and evaporated under vacuo. The crude mixture was purified by flash columin chromatography (hexane/EtOAc, $5: 1, \mathrm{v} / \mathrm{v})$ to afford pure $3(2.76 \mathrm{~g}$. $87 \%$ ) as an oil. $[\alpha]_{\mathrm{L}}=-4.3\left(c 2.00 . \mathrm{CHCl}_{3}\right)$ : IR (KRS-5) 2980 . $2970.2970,2350.1370 .1310,1290.1100,920 \mathrm{~cm}^{-1}$ : ${ }^{1} \mathrm{H}$ NMR $\left(500 \mathrm{MHz}, \mathrm{CDCl}_{3}\right)$ ò 1.37 (s. 3H), 1.42 (s. 3H). 1.43 (s. $\left.3 \mathrm{H}\right)$. 1.45 (s. $3 \mathrm{H}), 2.84-2.85$ (n. $2 \mathrm{H}), 3.22(\mathrm{dd}, 1 \mathrm{H} . J=3.5,6.8 \mathrm{~Hz})$. 3.85 (t. $1 \mathrm{H}, J=7.29 \mathrm{~Hz}$ ). 3.98 (dd. IH, $J=4.9 .8 .1 \mathrm{~Hz}$ ). 4.07.4.16 (m, 3H): ${ }^{13} \mathrm{C}$ NMR (125 MHz. $\left.\mathrm{CDCl}_{3}\right) \delta 110.4$, $110.1,79.2 .79 .1,77.1 .67 .8,52.3 .44 .6 .31 .3,27.4 .27 .1,27.0$. 25.6. Anal. Calcd for $\mathrm{C}_{12} \mathrm{H}_{2} \mathrm{O}_{5}: \mathrm{C} .59 .00: \mathrm{H}, 8.25$. Found: $\mathrm{C}$. $58.98 ;$ H. 8.24 .

General preparation of epoxy $5 a, 5 b$ and $5 c$ : Repuesentative procedure for the preparation of 1-(keoxy-1-cyclohexyl-3,4;5,6$O$-diisopropylidene- $D$-mannose (5a). To a solution of dried CuI (1.37 g. $7.2 \mathrm{mmol} .99 .99 \%)$ in ether (20 mL) was slowly added dropuise for 5 min cyclohexylmagnesium chloride ( $4 \mathrm{~mL}$. $8.0 \mathrm{mmol}$ ) at $-20{ }^{\circ} \mathrm{C}$. After stirring for $5 \mathrm{~min}$. compound 4 $(0.488$ g. $2.00 \mathrm{~mm}$ mol) dissolved in ether $(10 \mathrm{~mL})$ added for 5 min via cannula and stirred for $30 \mathrm{~min}$. To the reaction mixture added saturated $\mathrm{NH}_{4} \mathrm{Cl}$ solution ( $5 \mathrm{~nL}$ ) and stirred additional $20 \mathrm{~min}$. The reaction quenched with water $(30 \mathrm{~mL})$ and extracted with EtOAc $(30 \mathrm{~mL} \times 3)$. The organic layer washed with water $(30 \mathrm{~mL} \times 2)$ and brine and dried with anhy drous $\mathrm{MgSO}_{4}$. Filtration and removal of the solvent gave a dark yellow oil which was further purified by flash colunin clurontatography (hexane/EtOAc. $10 / 1$, w $/$ ) to afford pure 5 a $(1.67 \mathrm{~g} .70 \%$ ) as white solid.

$\operatorname{mp} 42-44^{\circ} \mathrm{C} .[\alpha]_{\mathrm{D}}=-3.6\left(c 3.00, \mathrm{CHCl}_{\mathrm{s}}\right)$ : IR (KBr) 3470 , $2980,2950,2920,2890,2850,1440,1350,1240,1220,1140$. $1070.860 \mathrm{~cm}^{-1} ;{ }^{1} \mathrm{H}$ NMR $\left(500 \mathrm{MHz}, \mathrm{CDCl}_{3}\right) \delta 0.85-0.88(\mathrm{~m}$, lH). $0.98-1.03(\mathrm{~m} . \mathrm{lH}) .1 .14-1.19(\mathrm{~m}, \mathrm{lH}) .1 .23-1.30(\mathrm{~m} . \mathrm{HH})$. $1.36(\mathrm{~s} .6 \mathrm{H}), 1.37$ (s, 3H). $1.39-1.42(\mathrm{~m}, 1 \mathrm{H}) .1 .4+(\mathrm{s} .3 \mathrm{H})$, $1.50-1.59$ (m. $2 \mathrm{H}) .1 .63-1.71(\mathrm{~m}, 5 \mathrm{H}) .1 .84-1.89(\mathrm{~m}, 1 \mathrm{H}), 3.11$ (s. $-\mathrm{OH}), 3.7 \mathrm{I}-3.76(\mathrm{~m}, 3 \mathrm{H}), 3.98$ (dd, IH, $J=5.7,8.44 \mathrm{~Hz}$ ). $4.14-4.07$ (n. $1 \mathrm{H}), 4.20(\mathrm{dd}, 1 \mathrm{H}, J=5.92 .8 .7 \mathrm{~Hz}):{ }^{13} \mathrm{C} \mathrm{NMR}$ $\left(\mathrm{I} 25 \mathrm{MHz}, \mathrm{CDCl}_{3}\right)$ oे $110.2,109.1,84.1,80.8,77.3,76.7,69.7$. $68.1 .34 .7 .33 .6,32.5 .26 .9,27.0 .26 .7 .26 .5,26.5 .26 .2,25.2$. Anal Calcd for $\mathrm{C}_{18} \mathrm{H}_{3} \mathrm{O}_{5}: \mathrm{C}, 65.82: \mathrm{H}, 9.82$. Found: C. 65.80 : H. 9.81 .

1-Deoxy-1-isopropỵl-3,4;5,6-O-(liisopropylidene- $D$-man- 
nose (5b). According to the general procedure with isopropylmagnesium chloride ( $9 \mathrm{~mL} .18 .1 \mathrm{mmol}$ ) and compound 4 (2.95 g. $12.1 \mathrm{mmol}$ ) in THF afforded pure compound $\mathbf{5 b}$ (2.81 g. $80 \%$ ) as a colorless oil. $\left.[\alpha]_{D}=-3.0(c) 3.00, \mathrm{CHCl}_{3}\right)$; IR (KRS-5) 3470, 2980, 2950, 2930, 2900, 2870, 1450, 1370. 1240. $1210,1150,1070,850 \mathrm{~cm}^{-1}:{ }^{l} \mathrm{HNMR}\left(500 \mathrm{MHz} . \mathrm{CDCl}_{3}\right)$ ô 0.94 (dd, $6 \mathrm{H}, J=6.6,15.2 \mathrm{~Hz}) .1 .34$ (s. $3 \mathrm{H}), 1.36(\mathrm{~s}, 3 \mathrm{H})$. $1.38(\mathrm{~s} .3 \mathrm{H}) .1 .41(5.3 \mathrm{H}), 1.42-1.47(\mathrm{~m} .2 \mathrm{H}) .1 .81-1.90(\mathrm{~m} . \mathrm{HH})$. $3.16(\mathrm{~s} .-\mathrm{OH}), 3.71-3.75(\mathrm{~m} .3 \mathrm{H}), 3.97-4.00(\mathrm{dd} . \mathrm{IH}, J=5.6$. $8.5 \mathrm{~Hz})+.05-4.06(\mathrm{~m} . \mathrm{lH}),+.19(\mathrm{dd}, 1 \mathrm{H}, J=6.1 .8 .5 \mathrm{~Hz}):{ }^{13} \mathrm{C}$ NMR $\left(125 \mathrm{MHz}_{2} \mathrm{CDCl}_{3}\right)$ ò $110.5,109.5,84.4 .81 .1,77.0$. $70.6,68.4,43.0,27.3,27.2,26.8,25.6,24.5,24.3,21.9$. Anal. Caled for $\mathrm{C}_{15} \mathrm{H}_{2} \mathrm{O}_{5}:$ C. $62.47 ; \mathrm{H} .9 .79$. Found: $\mathrm{C}, 62.45 ; \mathrm{H} .9 .78$.

1-Deoxy-1-methyl-3,4;5,6-O-diisopropylidene- $D$-mannose (5c). According to the general procedure with methylmagnesium bromide $(5.18 \mathrm{~mL} .15 .5 \mathrm{mmol})$ and compound 4 $(2.53 \mathrm{~g} .10 .+\mathrm{mmol})$ in $\mathrm{Et}_{2} \mathrm{O}$ afforded pure compound $5 \mathrm{c}(2.95$ g. $80 \%)$ as a colorless oil. $[\alpha]_{\mathrm{D}}=-2.23\left(c 2.00 . \mathrm{CHCl}_{3}\right)$ : IR (KRS-5) 3480, 2980, 2930, 2880, 1370, 1240, 1210, 1150. $1060.840 \mathrm{~cm}^{-1} ;{ }^{1} \mathrm{H}$ NMR $\left(500 \mathrm{MHz} . \mathrm{CDCl}_{3}\right)$ ò $1.02(\mathrm{t}, 3 \mathrm{H}, J$ $=7.3 \mathrm{~Hz}) .1 .23(\mathrm{~s} .3 \mathrm{H}) \cdot 1.2+(\mathrm{s} .3 \mathrm{H}) .1 .2+(\mathrm{s} .3 \mathrm{H}) .1 .25(\mathrm{~s} .3 \mathrm{H})$, $1.51-1.69(\mathrm{~m}, 2 \mathrm{H}), 1.73-1.80(\mathrm{~m}, \mathrm{lH}), 3.39(\mathrm{~m},-\mathrm{OH})$. 3.54-3.58 (m, 1H), 3.70-3.74 (m, 2H), $4.01(\mathrm{dd}, 1 \mathrm{H}, J=3.0$. $5.5 \mathrm{~Hz})+4.05-4.09(\mathrm{~m} . \mathrm{HH})+20(\mathrm{dd}, 1 \mathrm{H}, J=6.0 .8 .5 \mathrm{~Hz}):{ }^{13} \mathrm{C}$ NMR (125 MHz, $\left.\mathrm{CDCl}_{3}\right) \delta 110.20,109.12 .83 .12$. 81.11, 76.58. 73.35. 68.07. 26.91. 26.80. 26.44. 26.40. 25.18.9.41. Anal. Calcd for $\mathrm{C}_{13} \mathrm{H}_{24} \mathrm{O}_{5}$ : C. 59.98; H. 9.29. Found: C. 59.99; H, 9.28 .

General preparation of 7a, 7b and 7c: Representative procedure for the preparation of 1-cyclohexyl-1-deoxy-3,45,6$O$-diisopropylidene-2- $O$-methansulfonyl- $D$-mannose ( 7 a). To a solution of $5 \mathrm{a}(2.0 \mathrm{~g} .6 .09 \mathrm{mmol})$ in $\mathrm{CH}_{2} \mathrm{Cl}_{2}(20 \mathrm{~mL})$ was added dropwise $\mathrm{Et}_{3} \mathrm{~N}(0.2 \mathrm{~g} .1 .83 \mathrm{mmol})$ for $5 \mathrm{~min}$ at $0^{\circ} \mathrm{C}$. After stirring for $5 \mathrm{~min} . \mathrm{MsCl}(0.76 \mathrm{~g} .6 .70 \mathrm{mmol})$ was slowly added to the mixture. The reaction mixture stirred for $30 \mathrm{~min}$ at $\mathrm{rt}$. and then was quenched with saturated aqueous $\mathrm{NaHCO}_{3}(20 \mathrm{~mL})$. The reaction mixture extracted with EtOAc $(3 \times 30 \mathrm{~mL})$ and the organic layers were evaporated in vacuo. The combined organic extracts were dried with $\mathrm{Na}_{2} \mathrm{SO}_{4}$ and evaporated under vacuo. The crude mixture was purified by flash column chromatography (hexane/EtOAc. $10: 1, \mathrm{v} / \mathrm{v}$ ) to afford pure $7 \mathbf{a}(2 .+3$ g. 98\%) as an oil. $7 \mathrm{a}[\alpha]_{\mathrm{D}}=-5.69\left(c 3.00, \mathrm{CHCl}_{3}\right)$ : IR (KRS-5) $2980,2930,2880,1+50,1340,1210,1160,1060,910,840$ $\mathrm{cm}^{-1}$ : ${ }^{1} \mathrm{H}$ NMR $(500 \mathrm{MHz}, \mathrm{CDCl})$ ồ 0.81-0.88 (m, lH), 0.97$1.0+(\mathrm{m}, \mathrm{lH}) .1 .12-1.32(\mathrm{~m}, 4 \mathrm{H}) .1 .3+(\mathrm{s} .3 \mathrm{H}) .1 .37(\mathrm{~s} .3 \mathrm{H})$. 1.41 (s. $3 \mathrm{H}) .1 .41$ (s. $3 \mathrm{H}), 1.45-1.56(\mathrm{~m} .2 \mathrm{H}) .1 .64-1.87(\mathrm{~m}$. $6 \mathrm{H}), 3.06(\mathrm{~s} .3 \mathrm{H}), 3.74-3.80(\mathrm{~m} .1 \mathrm{H}) .3 .93(\mathrm{dd} .1 \mathrm{H}, J=5.6,8.6$ $\mathrm{Hz}) .403-4.07(\mathrm{~m} . \mathrm{IH}), 4.1+-4.30(\mathrm{~m} . \mathrm{lH})+4.33-4.38(\mathrm{~m}, \mathrm{lH})$. 5.01-5.04 (m, 1H): ${ }^{13} \mathrm{C}$ NMR (125MHz, CDCl $)$ ò 110.0 . $109.9 .81 .7 .79 .5 .78 .0 .77 .1 .68 .0,38.8 .37 .3 .33 .8,33.3 .32 .4$ $27.1,25.0,26.7 .26 .4 .26 .1,25.9 .25 .2$. Anal. Caled for $\mathrm{C}_{19 \mathrm{H}_{34} \mathrm{O}}=\mathrm{S}$ : C. 56.13 ; H. 8.43. Found: C. 56.12; H. 8.43.

7b: Yield : $99 \%:[\alpha]_{D}=+13.62\left(c+.00 . \mathrm{CHCl}_{3}\right)$ : $\mathbb{R}(\mathrm{KRS}-5)$ $2980.2870,1360,1460,1210,1170,1070.980 .920 \mathrm{~cm}^{-1} ;{ }^{l} \mathrm{H}$ NMR (500 MHz, $\mathrm{CDCl}_{3}$ ) ò 0.96 (dd. $6 \mathrm{H}, J=6.4,11.8 \mathrm{~Hz}$ ). 1.35 (s. $3 \mathrm{H}), 1.38(\mathrm{~s}, 3 \mathrm{H}), 1.42$ (s. $3 \mathrm{H}), 1.42$ (s. $3 \mathrm{H}), 1.14-1.49$ (m. $1 \mathrm{H}) .1 .80-1.85(\mathrm{~m} .2 \mathrm{H}) .3 .08(\mathrm{~s} .3 \mathrm{H}), 3.76-3.78(\mathrm{dd}, \mathrm{lH} . J$ $=7.3 .8 .5 \mathrm{~Hz}) .3 .95(\mathrm{dd} .1 \mathrm{H} . J=5.4 .8 .6 \mathrm{~Hz}) .4 .04-4.08(\mathrm{~m}$.
$1 \mathrm{H}) .4 .17(\mathrm{dd}, 1 \mathrm{H}, J=6.2 .8 .6 \mathrm{~Hz}) .4 .25(\mathrm{dd}, 1 \mathrm{H}, J=2.4 .7 .2$ $\mathrm{Hz})$ 4.99-5.03 (m, lH): ${ }^{13} \mathrm{C}$ NMR (125 $\left.\mathrm{MHz}^{1} \mathrm{CDCl}_{3}\right)$ oे 110.0 . $110.0,81.7 .80 .1,78.0 .77 .1,68.0 .38 .8 .38 .8,27.0 .27 .0,26.7$. 25.2, 24.0, 23.2. 21.6. Anal. Calcd for $\mathrm{C}_{16} \mathrm{H}_{30} \mathrm{O}-\mathrm{S}: \mathrm{C} .52 .44: \mathrm{H}$. 8.25. Found: C. $52.45:$ H. 8.26.

7c: Yield : $98 \%$ : $[\alpha]_{D}=-5.65\left(\mathrm{c} 2.00 . \mathrm{CHCl}_{3}\right) ; \mathrm{R}(\mathrm{KRS}-5)$ $2990,2940,2890,1460,1360,1240,1220,1180,1070,930$. $850 \mathrm{~cm}^{\cdot]}$ : ${ }^{1} \mathrm{H}$ NMR $\left(500 \mathrm{MHz}, \mathrm{CDCl}_{3}\right) \delta 1.02$ (t. $3 \mathrm{H} . J=7.8$ $\mathrm{Hz}$ ) 1.31 (s. $3 \mathrm{H}), 1.34$ (s. $3 \mathrm{H}), 1.38$ (s. $6 \mathrm{H}), 1.75-1.89(\mathrm{~m} .3 \mathrm{H})$. 3.04 (s. $3 \mathrm{H}$ ). 3.83 (dd, $1 \mathrm{H}, J=7.0 .8 .0 \mathrm{~Hz}) .3 .91$ (dd, $1 \mathrm{H}, J=$ $5.5 .8 .5 \mathrm{~Hz}) .4 .02-4.06(\mathrm{~m} .1 \mathrm{H}), 4.11$ (dd. $1 \mathrm{H}, J=6.0 .8 .5 \mathrm{~Hz})$, 4.17 (dd. IH. $J=3.5 .7 .0 \mathrm{~Hz}), 4.76-4.79(\mathrm{~m}, \mathrm{IH}):{ }^{15} \mathrm{C} \mathrm{NMR}$ (125 MHz. CDCl $)$; ô 110.t. 110.2.84.0.81.1. 78.6. 77.4, 68.0. 39.0. 27.5. 27.5. 26.9, 25.6. 23.7. 10.1. Anal. Calcd for $\mathrm{C}_{14} \mathrm{H}_{26} \mathrm{O}-\mathrm{S}: \mathrm{C}, 49.69:$ H. 7.74. Found: $\mathrm{C}, 48.70 ; \mathrm{H} .7 .76$.

General preparation of $8 \mathrm{a}, 8 \mathrm{~b}$ and $8 \mathrm{c}$ : Representative procedue for the preparation of 2-azido-1-cyclohexyl-1,2-dideoxy3,$4 ; 5,6-0$-diisopropylidene-D-glucose (8a). To a solution of 7 a $(2.0 \mathrm{~g} .6 .39 \mathrm{mmol})$ in $\mathrm{N}, \mathrm{k}$-dimethyl formamide $(20 \mathrm{~mL})$ was added $\mathrm{NaN}_{5}$ ( $3.20 \mathrm{~g} .32 \mathrm{mmol}, 5$ times excess) at rt. The reaction mixture stirred for $10 \mathrm{~h}$ at $80^{\circ} \mathrm{C}$ and then cooled down rt. and quenched with water $(30 \mathrm{~mL})$. The reaction mixture extracted with EtOAc $(3 \times 30 \mathrm{~mL})$ and the organic layers were evaporated in vacuo. The combined organic extracts were dried with $\mathrm{Na}_{2} \mathrm{SO}_{4}$ and evaporated under vacuo. The crude mixture was purified by flash column chromatography (hexane/EtOAc, $10: 1, \mathrm{v} / \mathrm{v})$ to afford pure $8 \mathrm{a}(1.97 \mathrm{~g} .87 \%$ ) as an oil. $[\alpha]_{D}=-6.69\left(\mathrm{c} 2.00 . \mathrm{CHCl}_{3}\right)$ : IR (KRS-5) 2990. 2920, 2850 , $2360.2110 \mathrm{~cm}^{-1}:{ }^{1} \mathrm{H}$ NMR $\left(500 \mathrm{MHz}, \mathrm{CDCl}_{2}\right) \delta 0.89-0.92(\mathrm{~m}$. lH). $0.97-1.00$ (m. 1H). 1.15-1.20 (n. 1H). $1.23-1.31$ (n. $2 \mathrm{H}$ ). $1.32(\mathrm{~s} .3 \mathrm{H}), 1.37$ (s. $3 \mathrm{H}), 1.39(\mathrm{~s} .3 \mathrm{H}), 1.47(\mathrm{~s} .3 \mathrm{H}) .1 .48-1.52$ (m, 2H). 1.66-1.85 (m, 6H). 3.28-3.32 (m, 1H). 3.91-4.03 (m, 4H). 3.13 (dd. $1 \mathrm{H}, J=5.64,8.33 \mathrm{~Hz}){ }^{1}{ }^{i} \mathrm{C}$ NMR $(125 \mathrm{MHz}$, $\left.\mathrm{CDCl}_{3}\right) \hat{\mathrm{o}} 110.1,109.8,83.3,78.0 .77 .4,67.9 .58 .2,38.5 .34 .5$, 33.8. 32.5. 27.2. 26.7. 26.5. 26.3. 26.1.25.3. Anal. Calcd for $\mathrm{C}_{18} \mathrm{H}_{21} \mathrm{~N}_{2} \mathrm{O}_{4}: \mathrm{C} .61 .17: \mathrm{H} .8 .84$. Found: C. 61.18: H. 8.85 .

8b: Yield : $88 \%$ : $[\alpha]_{D}=-25.49\left(\mathrm{c}+.00, \mathrm{CHCl}_{3}\right)$. IR $(\mathrm{KRS}-5)$ $2990,2960,2940,2870,2110,1470,1370,1250,1220,1070$. $850 \mathrm{~cm}^{-1}:{ }^{1} \mathrm{H}$ NMR $\left(500 \mathrm{MHz} . \mathrm{CDCl}_{3}\right)$ ô $0.96(\mathrm{~d}, 3 \mathrm{H} . J=6.5$ $\mathrm{Hz}$ ), 0.99 (d, $3 \mathrm{H}, J=6.44 \mathrm{~Hz}), 1.32$ (s. $3 \mathrm{H}$ ). 1.37 (s. $3 \mathrm{H}) .1 .39$ (s. $3 \mathrm{H}) 1.47$ (s. $3 \mathrm{H}), 1.47-1.49(\mathrm{~m}, \mathrm{lH}), 1.82-1.87(\mathrm{~m}, 2 \mathrm{H})$, 3.28-3.28 (n. $4 \mathrm{H}) .4 .13$ (dd. $1 \mathrm{H}, J=5.5,8.2 \mathrm{~Hz}$ ): ${ }^{13} \mathrm{C}$ NMR $\left(500 \mathrm{MHz}, \mathrm{CDCl}_{2}\right) \hat{o} \mathrm{l} 10.1,109.8,83.1 .78 .0 .77 .5,68.0 .58 .9$. 39.8. 27.2. 26.7. 26.7. 25.3. 25.1. 23.0. 21.8. Anal. Calcd for $\mathrm{C}_{15} \mathrm{H}_{2}: \mathrm{N}_{3} \mathrm{O}_{4}:$ C. 57.4: H, 8.68. Found: C. 57.48: H, 8.67.

8c: Yield : $85 \%:[\alpha]_{D}=-11.34$ (c $\left.3.00 . \mathrm{CHCl}_{2}\right)$ : IR (KRS-5) $2990.2930,2880.2100 .1380,1250.1220,1070.850 \mathrm{~cm}^{-1}:{ }^{l} \mathrm{H}$ NMR (500MHz, $\left.\mathrm{CDCl}_{3}\right) \delta 1.09$ (t. $3 \mathrm{H}, J=7.4 \mathrm{~Hz}$ ). 1.33 (s. $3 \mathrm{H}), 1.37$ (s. $3 \mathrm{H}) .1 .40$ (s. 3H), $1.46(\mathrm{~s}, 3 \mathrm{H}), 1.74-1.79(\mathrm{~m}, 1 \mathrm{H})$, $1.86-1.92(\mathrm{~m}, 1 \mathrm{H}), 3.13-3.14(\mathrm{~m}, \mathrm{lH}), 3.95-4.12(\mathrm{~m}, 4 \mathrm{H})$. $4.13-4.15$ (dd, $1 \mathrm{H}, J=5.7 .8 .3 \mathrm{~Hz}) ;{ }^{15} \mathrm{C} \mathrm{NMR}(125 \mathrm{MHz}$, $\mathrm{CDCl}_{3}$ ) ô $110.0 .109 .8 .82 .5,82.0 .79 .0,78.0 .77 .4,68.0 .67 .6$, $65.6 .62 .6,27.5,27.2,26.8,26.7,26.5,25.3 .24 .4,23.3 .11 .1$. 11.0. Anal. Calcd for $\mathrm{C}_{33} \mathrm{H}_{2} \mathrm{~N}_{3} \mathrm{O}_{4}: \mathrm{C}, 54.72 ; \mathrm{H}, 8.12$. Found: $\mathrm{C}$, 54.73 : H. 8.13.

General preparation of $9 \mathrm{a}, 9 \mathrm{~b}$ and $9 \mathrm{c}$ : Representative procedure for the meparation of 2-amino-1,2-dideoxy-1-cyclohexyl3,$4 ; 5,6$-O-diiso-propylidene- $D$-glucose (9a). To a solution of 
$8 \mathrm{a}(1 .+\mathrm{g}, 4.25 \mathrm{mmol})$ in $\mathrm{MeOH}(20 \mathrm{~mL})$ was added catalytic amount of $10 \% \mathrm{Pd} / \mathrm{C}$ and the reaction mixture stirred for $30 \mathrm{~h}$ at rt. Reaction mixture filtered by glass funnel which was padded with celite. The solvent was evaporated in vacuo. The crude mixture was purified by flash column chromatography (hexane/EtOAc, 1:2, $/ \%$ ) to afford pure $9 \mathrm{a}(1.32 \mathrm{~g} .95 \%$ ) as an oil. $9 \mathrm{a}:[\alpha]_{D}=-13.26\left(\mathrm{c} 2.00, \mathrm{CHCl}_{3}\right)$ : IR $(\mathrm{KRS}-5) 3390.3330$. 2990, 2920. 2850.1370, 1240.1210.1070,850 $\mathrm{cm}^{-1} ;{ }^{1} \mathrm{H}$ NMR $\left(500 \mathrm{MHz}, \mathrm{CDCl}_{3}\right)$ ò $0.83-0.86(\mathrm{~m}, \mathrm{lH}), 0.95-0.97(\mathrm{~m}, \mathrm{lH})$ $1.1+-1.19(\mathrm{~m} . \mathrm{lH}) .1 .23-1.29(\mathrm{~m} .5 \mathrm{H}) .1 .34-1.40(\mathrm{~m} . \mathrm{lH}) .1 .34$ $(\mathrm{s}, 3 \mathrm{H}), 1.36(\mathrm{~s} .3 \mathrm{H}), 1.40(\mathrm{~s}, 3 \mathrm{H}) .1 .4 \mathrm{l}(\mathrm{s} .3 \mathrm{H}) .1 .46-1.52(\mathrm{~m}$, lH) $1.64-1.7 \mathrm{l}(\mathrm{m},+\mathrm{H}) .1 .77-\mathrm{l} .89(\mathrm{~m}, \mathrm{lH}) .2 .90-2.9+(\mathrm{m}, \mathrm{lH})$. $3.80($ dd. $1 \mathrm{H} . J=3.85,6.79 \mathrm{~Hz}) .3 .85(\mathrm{dd}, 1 \mathrm{H} . J=6.8 .8 .0 \mathrm{~Hz})$, 3.96 (dd $1 \mathrm{H}, J=5.1,8.4 \mathrm{~Hz}), 4.03-4.07(\mathrm{~m}, \mathrm{lH})+.13$ (dd. $1 \mathrm{H}$. $J=6.16 .8 .38 \mathrm{~Hz}) ;{ }^{13} \mathrm{C}$ NMR $\left(125 \mathrm{MHz}^{\mathrm{CDCl}} \mathrm{CDC}_{3}\right) \delta 109.6$. 109.2.84.4. 78.4, 77.4. 67.8. 49.3, 43.2. 34.4, 34.2, 32.6. 27.5, $27.4,26.7,26.7,26.4,26.2,25.3$. Anal Calcd for $\mathrm{C}_{18} \mathrm{H}_{33} \mathrm{NO}_{4}$ : C. 66.05 ; H. $10.09:$ N. 4.28. Found: C, 66.04; H. 10.07: N. +25 .

9b: $[\alpha]_{\mathrm{D}}=-19.4\left(\mathrm{c} 2.00, \mathrm{CHCl}_{3}\right) ; \mathrm{R}(\mathrm{KRS}-5) 3480,3400$. $3060.2960 .2930,2850,2340.1710,1610,1+50,1300,920$. $730 \mathrm{~cm}^{-1}:{ }^{1} \mathrm{H} \mathrm{NMR}\left(500 \mathrm{MHz}, \mathrm{CDCl}_{3}\right) \delta 0.90$ (d. $3 \mathrm{H}, J=6.54$ $\mathrm{Hz}), 0.94(\mathrm{~d}, 3 \mathrm{H}, J=6.62 \mathrm{~Hz}), 1.29-1.40(\mathrm{~m}, 5 \mathrm{H}), 1.34(\mathrm{~s}, 3 \mathrm{H})$, $1.36(\mathrm{~s} .3 \mathrm{H}), 1.40(\mathrm{~s} .6 \mathrm{H}), 1.77-1.83(\mathrm{~m} . \mathrm{lH}) .2 .88-2.91(\mathrm{~m}$. $1 \mathrm{H}), 3.82$ (dd. $1 \mathrm{H}, J=3.5,6.9 \mathrm{~Hz}) .3 .88$ (dd. $1 \mathrm{H}, J=6.9 .7 .9$ Hz) 3.96 (dd. IH. $J=5.05 .8 .+\mathrm{Hz}) .+03-4.07(\mathrm{~m} . \mathrm{lH}) .4 .13$ (dd. $1 \mathrm{H} . J=6.1,8.4 \mathrm{~Hz}) ;{ }^{13} \mathrm{C} \mathrm{NMR}\left(125 \mathrm{MHz} . \mathrm{CDCl}_{3}\right)$ ò 109.6 , 109.2.84.1. 78.3, 77.4.67.8. 49.9, 44.8. 27.4.27.3, 26.7.25.3,

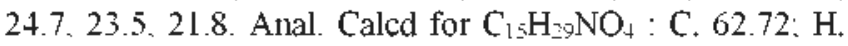
$10.10 ; \mathrm{N}, 4.87$. Found: C. $62.71 ; \mathrm{H}, 10.08: \mathrm{N}, 4.85$.

$9 \mathrm{c}:[\alpha]_{\mathrm{D}}=+8.28\left(\mathrm{c} 2.00, \mathrm{CHCl}_{3}\right):$ IR (KRS-5) 3380.3390 . 2980.2930.2880.2360.1460.1380. 1250. 1220. 1160, 1070, $850 \mathrm{~cm}^{-l}:{ }^{\mathrm{H}} \mathrm{H}$ NMR $\left(500 \mathrm{MHz}, \mathrm{CDCl}_{3}\right)$ ò $1.00(\mathrm{t} .3 \mathrm{H}, J=7.4$ $\mathrm{Hz}, 1.27$ (s. 2H) $1.3+(\mathrm{s} .3 \mathrm{H}) \cdot 1.36$ (s. $3 \mathrm{H}), 1.40$ (s. $3 \mathrm{H}$ ), $1.34-1.39$ (m, 1H). 1.58-1.63 (m. 1H). 2.70-2.73 (m. 1H), $3.85-3.91(\mathrm{~m} .2 \mathrm{H}) .3 .96(\mathrm{dd} . \mathrm{IH} . J=5.20 .8 .35 \mathrm{~Hz})+4.03-4.07$ $(\mathrm{m}, \mathrm{lH}) .4 .13$ (dd. $1 \mathrm{H} . J=6.2,8.3 \mathrm{~Hz}):{ }^{13} \mathrm{C}$ NMR $(125 \mathrm{MHz}$. $\left.\mathrm{CDCl}_{3}\right) \delta 110.0 .109 .4 .83 .9 .78 .6 .68 .1,53.7 .31 .2,28.7 .27 .7$. 27.6, 27.0, 25.7, 11.2. Anal. Calcd for $\mathrm{C}_{13} \mathrm{H}_{35} \mathrm{NO}_{4}: \mathrm{C}, 60.23$ : H, $9.65 ; \mathrm{N}, 5.41$. Found: C. $60.22 ; \mathrm{H}, 9.66 ; \mathrm{N} .5 .44$.

Genenal preparation of $10 \mathrm{a}, 10 \mathrm{~b}$ and $10 \mathrm{c}$ : Representative procedure for the preparation of 1-cyclohexyl-1,2-dideoxy3,$4 ; 5,6-O$-diisopropylidene-2-[(9-phenylfluoren-9-yl)amino)$D$-glucose (10a). To a solution of $9 \mathrm{a}(0.9 \mathrm{~g}, 3.05 \mathrm{mmol}) \mathrm{in}$ $\mathrm{CH}_{2} \mathrm{Cl}_{2}(10 \mathrm{~mL})$ was added $\mathrm{Pb}\left(\mathrm{NO}_{3}\right)_{2}(0.94 \mathrm{~g} .4 .58 \mathrm{mmol})$. 9-bromo-9-phenylfluorene $(1.47 \mathrm{~g} .4 .58 \mathrm{mmol})$ and $\mathrm{Et}_{3} \mathrm{~N}$ $(0.93 \mathrm{~g}, 9.16 \mathrm{mmol})$ at $\mathrm{rt}$. The reaction mixture stirred for 30 min at $\mathrm{rt}$. and quenched with water $(30 \mathrm{~mL})$. The reaction mixture extracted with $\mathrm{CH}_{2} \mathrm{Cl}_{2}(3 \times 20 \mathrm{~mL})$ and the organic layers were evaporated in vacuo. The combined organic extracts were dried with $\mathrm{Na}_{2} \mathrm{SO}_{4}$ and evaporated under vacuo. The crude mixture was purified by flash column chromatography (hexane/EtOAc. $10: 1 . v / v)$ to afford pure $10 \mathrm{a}(1.51 \mathrm{~g} .87 \%$ ) as an oil.

10a: $[\alpha]_{D}=+7.44\left(\mathrm{c} 2.00 . \mathrm{CHCl}_{3}\right)$ : IR (KRS-5) 3430. 3330. $3060.2990 .2920 .2850 .1720,1450,1370,1250,1210,1060$, $850 \mathrm{~cm}^{-1}$ : ${ }^{l} \mathrm{H}$ NMR $\left(500 \mathrm{MHz}, \mathrm{CDCl}_{3}\right)$ ò $0.28-0.33(\mathrm{~m}, \mathrm{lH})$. $0.39-0.45(\mathrm{~m} . \mathrm{lH}), 0.88-1.17(\mathrm{~m} .7 \mathrm{H}) .1 .19-1.24(\mathrm{~m} .5 \mathrm{H}) .1 .25$ (s. 3H), 1.13 (s. 3H), 1.14-1.44 (n. +H), 1.50-1.55 (m. 3H), $2.34-2.45(\mathrm{~m} . \mathrm{lH}) .2 .63(\mathrm{~d}, \mathrm{lH}, J=8.7 \mathrm{~Hz}) .3 .64-3.69(\mathrm{~m} .2 \mathrm{H})$. $3.90-4.03$ (n. $3 \mathrm{H}) .7 .07-7.21$ (m. $5 \mathrm{H}), 7.28-7.39$ (m. $4 \mathrm{H})$, $7.43-7.45$ (n. $2 \mathrm{H}$ ). 7.67 (d. $2 \mathrm{H} . J=7.5 \mathrm{~Hz}$ ); ${ }^{13} \mathrm{C} \mathrm{NMR}$ $\left(125 \mathrm{MHz}, \mathrm{CDCl}_{3}\right)$ ò 151.0,150.5, 145.9, 140.6, 140.5, 128.1, $128.1,127.7$. 127.5, 126.9, 126.5. 126.3, 126.1, 125.9. 125.8, $120.0,119.8,109.3,108.4,80.9,77.3,76.8,72.4,67.5,49.3$. $41.5 .33 .7 .33 .3,32.8 .30 .9,27.3 .26 .8,26.6,26.4 .26 .2,26.1$. 25.3. 13.8. Anal. Calcd for $\mathrm{C}_{36} \mathrm{H}_{45} \mathrm{NO}_{4}: \mathrm{C}, 77.80: \mathrm{H}, 8.16: \mathrm{N}$. 2.52. Found: C. 77.83: H, 8.13: N. 2.55.

10b: Yield : $85 \%$ : $[\alpha]_{\mathrm{D}}=+3.84$ (c $2.00, \mathrm{CHCl}_{3}$ ): IR (KRS-5) 3330.3060 .2980 .2930 .2870 .1740 .1450 .1380 .1250 .1210$. $1160,1070.850,740 \mathrm{~cm}^{-1} ;{ }^{1} \mathrm{H}$ NMR $\left(500 \mathrm{MHz}, \mathrm{CDCl}_{3}\right)$ ô 0.38 (d. $3 \mathrm{H}, J=6.5 \mathrm{~Hz}), 0.45$ (d. $3 \mathrm{H} . J=6.5 \mathrm{~Hz}), 0.83-0.87(\mathrm{~m} . \mathrm{lH})$. $1.17-1.2+$ (n. 1H), 1.22 (s, 3H). 1.26 (s, 3H). 1.30 (s. $3 \mathrm{H})$, $1.34-1.4+$ (n. 1H), $1.46(\mathrm{~s}, 3 \mathrm{H}) .2 .32$ (s, 1H). $2.56(\mathrm{~s} .1 \mathrm{H})$, $3.67-3.71(\mathrm{~m}, \mathrm{lH}), 3.88-3.92(\mathrm{~m}, \mathrm{lH}), 4.0 \mathrm{l}-4.03(\mathrm{~m}, \mathrm{lH})$. $7.16-7.35(\mathrm{~m} .9 \mathrm{H}) .7 .45(\mathrm{~d}, 2 \mathrm{H}, J=7.0 \mathrm{~Hz}) .7 .66(\mathrm{~d}, 2 \mathrm{H}, J=7.4$ $\mathrm{Hz}):{ }^{13} \mathrm{C} \mathrm{NMR}\left(125 \mathrm{MHz}, \mathrm{CDCl}_{2}\right) \delta 151.2,150.4,146.0,140.6$. $140.5,128.5 .128 .2,128.1,127.7 .127 .6,126.9,126.3 .125 .9$, $125.4 .120 .1 .119 .9,119.8,109.3 .108 .5,80.9 .77 .5,77.0 .72 .4$. $67.5 .50 .2,43.1 .27 .3 .26 .8 .26 .4 .25 .3 .24 .4 .22 .9 .21 .7$. Anal. Calcd for $\mathrm{C}_{33} \mathrm{H}_{41} \mathrm{NO}_{4}$ : C. 76.86; H. 8.01: N, 2.72 . Found: $\mathrm{C}$, 76.83: H. 8.03: N. 2.75 .

10c: Yield : $89 \% ;[\alpha]_{D}=+8.52\left(\mathrm{c} 2.00 . \mathrm{CHCl}_{3}\right)$; IR $(\mathrm{KRS}-5)$ 3420.3330 .3040 .2987 .2920 .2850 .1715 .1440 .1350 .1250 . 1210. $1060,870 \mathrm{~cm}^{-1} ;{ }^{1} \mathrm{H} \mathrm{NMR}\left(500 \mathrm{MHz}, \mathrm{CDCl}_{2}\right) \delta 0.64(\mathrm{t}$, $3 \mathrm{H} . J=7.4 \mathrm{~Hz}), 1.03-1.07(\mathrm{~m}, 1 \mathrm{H}) .1 .14-1.17(\mathrm{~m}, 1 \mathrm{H}) .1 .19(\mathrm{~s}$. $3 \mathrm{H}) .1 .27(\mathrm{~s} .3 \mathrm{H}), 1.32(\mathrm{~s} .3 \mathrm{H}), 1.39(\mathrm{~s}, 3 \mathrm{H}), 2.25(\mathrm{~s} .1 \mathrm{H}), 2.27$ (s. $1 \mathrm{H}) .3 .68$ (dd. $1 \mathrm{H} . J=2.7,7.4 \mathrm{~Hz}), 3.75$ (dd. $1 \mathrm{H}, J=6.9 .8 .1$ Hz) $3.89-3.93(\mathrm{~m}, \mathrm{lH}) .3 .95-3.98(\mathrm{~m} . \mathrm{lH}),+0 \mathrm{l}-4.04(\mathrm{~m}, \mathrm{lH})$; ${ }^{13} \mathrm{C}$ NMR $\left(125 \mathrm{MHz} . \mathrm{CDCl}_{3}\right)$ ô $148.5 .148 .4,143.3,1+1.6$, $140.2,129.0,128.9,128.8,128.1,128.0,127.6,126.2,125.9$. $125.3 .120 .5,120.2 .107 .7 .81 .5,76.1 .73 .0 .72 .0 .64 .4,54.1$, $26.8,26.3,23.3,11.5$. Anal. Calcd for $\mathrm{C}_{31} \mathrm{H}_{3}: \mathrm{NO}_{4}: \mathrm{C}, 76.36 \mathrm{H}$. 7.65: N. 2.87. Found: C. 76.37: H. 7.63: N. 2.87.

General preparation of $11 \mathrm{~d}, 11 \mathrm{~b}$ and $11 \mathrm{c}$ : Representative procedure for the preparation of 1-cyclohexyl-1,2-dideoxy3,4-O-isopropylidene-2-[(9-pheny lfluo ren-9-y]) amino]$D$-lyxitol (11a). To a solution of $10 \mathrm{a}(1.17 \mathrm{~g} .2 .11 \mathrm{nmol})$ in $\mathrm{CH}_{2} \mathrm{Cl}_{2}(5 \mathrm{~mL})$ was added acetic $\operatorname{acid}(70 \%, 35 \mathrm{~mL})$ at $\mathrm{rt}$. The reaction mixture stirred for 24 hat $r t$ and then added water $(30$ $\mathrm{mL})$. The reaction mixture extracted with $\mathrm{CH}_{2} \mathrm{Cl}_{2}(3 \times 30 \mathrm{~mL})$. The combined organic extracts were dried with $\mathrm{Na}_{2} \mathrm{SO}_{4}$ and evaporated under vacuo. The crude mixture was purified by flash column chromatography (hexane/EtOAc. $10: 1 . \mathrm{v} / \mathrm{v}$ ) to afford pure $11 \mathrm{a}(0.98 \mathrm{~g}, 90 \%)$ as an oil

11a: $[\alpha]_{\mathrm{L}}=+29.26\left(\mathrm{c} 2.00 . \mathrm{CHCl}_{3}\right): \mathbb{R}(\mathrm{KRS}-5) 3440,3290$. $3060.2980,2930,2850,1720.1450 .1370 .1250,1070.740$ $\mathrm{cm}^{-1}:{ }^{1} \mathrm{H}$ NMR $\left(500 \mathrm{MHz}, \mathrm{CDCl}_{2}\right) \delta 0.38-0.4 \mathrm{I}(\mathrm{m} . \mathrm{lH})$. $0.75-0.78(\mathrm{~m}, 1 \mathrm{H}), 0.88-0.92(\mathrm{~m}, 1 \mathrm{H}), 0.94(\mathrm{~s}, 3 \mathrm{H}), 0.97-1.03$ $(\mathrm{m}, 2 \mathrm{H}), 1.04-1.16(\mathrm{~m}, 3 \mathrm{H}), 1.21(\mathrm{~s} .3 \mathrm{H}) .1 .2+-1.27(\mathrm{~m}, \mathrm{H})$, $1.36-\mathrm{l} .40(\mathrm{~m}, \mathrm{lH}), 1.42-1.46(\mathrm{~m}, 2 \mathrm{H}), 1.55-1.63(\mathrm{~m}, 2 \mathrm{H}) .2 .03$ (s, 1H), 2.54-2.57 (m. 1H). 3.12 (dd, $1 \mathrm{H}, J=3.2,11.7 \mathrm{~Hz}$ ), $3.47-3.50(\mathrm{~m}, \mathrm{lH}) .3 .67-3.74(\mathrm{~m}, 2 \mathrm{H}) .3 .35(\mathrm{dd}, \mathrm{lH}, J=4.0$. $11.2 \mathrm{~Hz}), 7.19-7.30(\mathrm{~m}, 7 \mathrm{H}), 7.31-7.36(\mathrm{~m}, 2 \mathrm{H}), 7.39-7.42(\mathrm{~m}$. lH). $7.46(\mathrm{~d}, \mathrm{lH}, J=7.5 \mathrm{~Hz}) .7 .74$ (d. $1 \mathrm{H}, J=7.6 \mathrm{~Hz}):{ }^{13} \mathrm{C}$ $\mathrm{NMR}\left(125 \mathrm{MHz}, \mathrm{CDCl}_{3}\right) \hat{o} \mathrm{l}+8.6,148.5,143.2 .141 .6,140.1$. 
129.0. 128.9. 128.8, 128.2. 128.1, 127.6, 126.3. 125.9. 124.9, $120.5,120.2,107.7,81.4 .76 .0 .73 .1,72.2 .64 .8,60.4,49.1$. $38.9 .34 .5 .33 .1,32.2,26.8,26.4,26.3,25.9 .14 .2$. Anal Calcd for $\mathrm{C}_{33} \mathrm{H}_{41} \mathrm{NO}_{4}:$ C. 76.86 H. $8.01 ;$ N. 2.72 . Found: C. $76.84: \mathrm{H}$. 8.00: N. 2.70 .

11b: Yield : $91 \%:[\alpha]_{\mathrm{D}}=+24.52\left(c 2.00 . \mathrm{CHCl}_{3}\right): \mathbb{R}(\mathrm{KRS}-5)$ $3410,3290,3060,2980,2950,2930,2870,2780,2250,1450$. 1370. 1240, $1070,910,740 \mathrm{~cm}^{-1}:{ }^{l} \mathrm{H} N \mathrm{NR}\left(500 \mathrm{MHz}, \mathrm{CDCl}_{3}\right)$ $\hat{o} 0.33$ (d. IH. $J=6.3 \mathrm{~Hz}), 0.73$ (d. IH. $J=6.5 \mathrm{~Hz}), 0.96$ (s. $3 \mathrm{H})$, $1.09-1.19(\mathrm{~m} .2 \mathrm{H}), 1.23-1.27(\mathrm{~m} .4 \mathrm{H}) .1 .33-1.39(\mathrm{~m} .1 \mathrm{H})$. $1.46-1.49(\mathrm{~m} .1 \mathrm{H}), 2.03(\mathrm{~s}, 1 \mathrm{H}) .2 .50-2.53(\mathrm{~m} .1 \mathrm{H}), 3.18$ (dd. IH. $J=3.2 .8 .5 \mathrm{~Hz}) .3 .48-3.52(\mathrm{~m} .1 \mathrm{H}) .3 .70-3.75(\mathrm{~m} . \mathrm{lH})$. $3.85(\mathrm{dd}, 1 \mathrm{H}, J=4.0,11.2 \mathrm{~Hz}) .7 .11-7.31(\mathrm{~m} .8 \mathrm{H}), 7.34-7.36$ (m. 2H) . 7.39-7.42 (m. IH). 7.45-7.49 (m. IH). 7.70-7.74 (m. $2 \mathrm{H}):{ }^{13} \mathrm{C} \mathrm{NMR}\left(125 \mathrm{MHz}, \mathrm{CDCl}_{3}\right)$ oे $148.7,148.5,143.2$, 141.6. 140.2. 129.0, 128.9. 128.7, 128.3, 128.0. 127.6. 126.4, $125.9 .125 .0,120.5,120.2,107.7,81.5,76.0,73.0 .72 .3 .64 .8$. $50.0,40.6,26.8 .26 .3 .24 .0,23.7 .21 .6$. Anal. Calcd for $\mathrm{C}_{31} \mathrm{H}_{3}: \mathrm{NO}_{4}:$ C. $75.76: \mathrm{H}, 7.84:$ N. 2.94. Found: C. 75.73: H. 7.83: N, 2.95

11c: Yield : $89 \%:[\alpha]_{\mathrm{D}}=+25.22\left(\mathrm{c} 2.00 . \mathrm{CHCl}_{3}\right)$ : IR (KRS-5) $3430,3310,3060,2980,2930,2870,1730,1600,1450,1380$. $1250.1070 \mathrm{~cm}^{-1} ;{ }^{1} \mathrm{H}$ NMR $\left(500 \mathrm{MHz} . \mathrm{CDCl}_{3}\right)$ o $0.73(\mathrm{t}, 3 \mathrm{H}, J$ $=7.5 \mathrm{~Hz}) .0 .96(\mathrm{~s} .3 \mathrm{H}), 1.2 \mathrm{I}-\mathrm{L} .27(\mathrm{~m} .2 \mathrm{H}), 1.22(\mathrm{~s} .3 \mathrm{H}) .1 .66$ $-1.71(\mathrm{~m} .1 \mathrm{H}), 2.03$ (s. $1 \mathrm{H}), 2.37-2.40(\mathrm{~m}, \mathrm{lH}), 3.15$ (dd, $1 \mathrm{H}$, $J=3.3 .8 .6 \mathrm{~Hz}) .3 .48-3.50(\mathrm{~m} . \mathrm{IH}) .3 .69-3.73(\mathrm{~m} .2 \mathrm{H}) .3 .85$ (dd. 1H. $J=3.9,11.1 \mathrm{~Hz}) .7 .19-7.31(\mathrm{~m} .7 \mathrm{H}) .7 .33-7.36(\mathrm{~m}$. lH), $7.39-7.42(\mathrm{~m}, \mathrm{lH}) .7 .47$ (d, $1 \mathrm{H} . J=7.6 \mathrm{~Hz}) .7 .71$ (dd. $2 \mathrm{H}$, $J=7.5 .17 .5 \mathrm{~Hz}):{ }^{i ?} \mathrm{C} \mathrm{NMR}\left(125 \mathrm{MHz}, \mathrm{CDCl}_{3}\right) \delta 148.5 .148 .4$. $143.3 .141 .6,140.2,129.0 .128 .9 .128 .8,128.1,128.0,127.6$, $126.2 .125 .9,125.3,120.3,120.2,107.7,81.5,76.1,73.0$. 72.0, 64.4. 54.1, 26.8, 26.3. 23.3, 11.5. Anal Caled for $\mathrm{C}_{28} \mathrm{H}_{33} \mathrm{NO}_{4}:$ C. $75.14: \mathrm{H}, 7.43:$ N. 3.13. Found: C. 75.12: H. 7.41: N. 3.10

General preparation of $12 \mathrm{a}, 12 \mathrm{~b}$ and $12 \mathrm{c}$ : Representative procedure for the preparation of 1-cyclohexyl-1,2-dideoxy3,4-0-isopmopylidene-2-[(9-phenylfluoren-9-y])amino]D-xylitol (12a). To a solution of 11a $(0.79 \mathrm{~g} .1 .53 \mathrm{mmol})$ in $\mathrm{H}_{2} \mathrm{O} / \mathrm{EtOH}=(1: 2,30 \mathrm{~mL})$ was added $\mathrm{NaIO}_{4}(0.49 \mathrm{~g}, 2.27$ mmol) at rt. The reaction mixture stirred for $2 \mathrm{~h}$ at rt. and then added $\mathrm{NaBH}_{4}(7.45 \mathrm{mg} .1 .97 \mathrm{mmol})$ and stirred for 15 min. EtOH in the reaction bottle was evaporated and the reaction mixture extracted with $\mathrm{CH}_{2} \mathrm{Cl}_{2}(3 \times 30 \mathrm{~mL})$. The combined organic extracts were dried with $\mathrm{Na}_{2} \mathrm{SO}_{4}$ and evaporated under vacuo. The cnide mixture was purified by flash column chromatography (hexane/EtOAc. $3: 1$. v/v) to afford pure $12 \mathrm{a}(0.67 \mathrm{~g}, 90 \%)$ as an oil.

12a: $[\alpha]_{D}=+18.51\left(c 2.00, \mathrm{CHCl}_{3}\right): \mathbb{R}(\mathrm{KRS}-5) 3+40.3060$. $3030.2990 .2980 .2850,1590,1370,1220.1150 .1050,930$, $740 \mathrm{~cm}^{-1}:{ }^{1} \mathrm{H} \mathrm{NMR}\left(500 \mathrm{MHz}, \mathrm{CDCl}_{3}\right)$ ò 0.38-0.45 (m. $\left.\mathrm{lH}\right)$. $0.60-68(\mathrm{~m} .1 \mathrm{H}) .0 .79-0.90(\mathrm{~m}, 2 \mathrm{H}), 0.96-1.03(\mathrm{~m} .2 \mathrm{H}) 1.04$ $(\mathrm{s}, 3 \mathrm{H}), 1.00-1.12(\mathrm{~m}, 3 \mathrm{H}), 1.24-1.29(\mathrm{~m}, \mathrm{H}), 1.28(\mathrm{~s}, 3 \mathrm{H})$. $1.30-1.58(\mathrm{~m} .2 \mathrm{H}), 1.55-\mathrm{l} .6 \mathrm{l}(\mathrm{m} . \mathrm{lH}), 2.30(\mathrm{~s} . \mathrm{lH}), 2.46-2.48$ $(\mathrm{m}, 1 \mathrm{H}), 3.15$ (dd, $1 \mathrm{H} . J=3.1 .8 .6 \mathrm{~Hz}) .3 .48(\mathrm{dd} .1 \mathrm{H}, J=8.3$. $10.7 \mathrm{~Hz}) .3 .71-3.74(\mathrm{~m}, \mathrm{lH}) .3 .94-3.98(\mathrm{~m}, \mathrm{lH}) .7 .22-7.48(\mathrm{~m}$. $11 \mathrm{H}) .7 .68-7.73(\mathrm{~m}, 2 \mathrm{H}) ;{ }^{13} \mathrm{C} \mathrm{NMR}\left(125 \mathrm{MHz}, \mathrm{CDCl}_{3}\right)$ ò $149.3 .148 .9,143.8,1+1.5,140.1,128.8,128.7,128.6,128.1$. $127.9 .127 .5,126.3 .126 .0,125.1,120.4 .120 .2,107.6,81.5$.
75.7. 73.0.63.0.49.0.39.5, 34.0.33.3. 32.6, 27.0. 26.5. 26.4. 26.3. 25.9. Anal. Calcd for $\mathrm{C}_{32} \mathrm{H}_{39} \mathrm{NO}_{3}$ : C. $79.14 \mathrm{H} .8 .09 \mathrm{~N}$. 2.88. Found: C, $79.12 \mathrm{H}, 8.07$ N. 2.85 .

12b: Yield : $79 \%:[\alpha]_{\mathrm{L}}=+20.32\left(\mathrm{c} 2.00, \mathrm{CHCl}_{3}\right)$; $\mathbb{R}(\mathrm{KRS}-5)$ $3460,3290,3060,2980,2950,2870,1450,1370,1240,1170$. $1070.910,730 \mathrm{~cm}^{-1}:{ }^{1} \mathrm{H} \mathrm{NMR}\left(500 \mathrm{MHz}, \mathrm{CDCl}_{3}\right)$ ô $0.43(\mathrm{~d}$, $3 \mathrm{H}, J=6.1 \mathrm{~Hz}), 0.62(\mathrm{~d} .3 \mathrm{H}, J=6.3 \mathrm{~Hz}), 1.07-1.11(\mathrm{~m}, \mathrm{lH})$, 1.17 (s. $3 \mathrm{H}$ ). 1.37 (s, $3 \mathrm{H}$ ), $1.39-1.49$ (n. $2 \mathrm{H}$ ). $2.42-2.45$ (m. lH). 3.29 (dd. IH, $J=2.9 .8 .6 \mathrm{~Hz}$ ). $3.5+$ (dd. $\mathrm{IH}, J=7.6 .10 .9$ Hz) 3.70 (dd. $1 \mathrm{H}, J=4.2 .10 .9 \mathrm{~Hz}), 4.04-4.09$ (m. lH), 7.23-7.45 (n. 11H). 7.72-7.77 (m, 2H); ${ }^{13} \mathrm{C} \mathrm{NMR}(125 \mathrm{MHz}$, $\left.\mathrm{CDCl}_{3}\right)$ o $149.5,148.9,144.0,141.4,140.2,128.8,128.7$. $128.5,128.1$. 127.8, 127.4, 126.2. 126.0, 125.2, 120.3. 120.1, $107.6 .81 .0 .75 .9 .72 .9 .62 .8,49.6 .41 .3 .27 .0 .26 .6 .24 .1 .22 .9$. 22.1. Anal. Calcd for $\mathrm{C}_{29} \mathrm{H}_{35} \mathrm{NO}_{3}:$ C, 78.17 H. 7.92 N. 3.14 . Found: C. 78.13 H. 7.93 N, 3.15 .

12c: Yield : 78\%: $[\alpha]_{D}=+35.28\left(c 2.00 . \mathrm{CHCl}_{3}\right): \operatorname{RR}(\mathrm{KRS}-5)$ 3460.3340 .3230 .3060 .2980 .2930 .2870 .1740 .1450 .1370$. $1240.1170,1050 \mathrm{~cm}^{-1}:{ }^{1} \mathrm{H} \operatorname{NMR}\left(500 \mathrm{MHz}, \mathrm{CDCl}_{3}\right) \delta \delta 0.66(\mathrm{t}$. $3 \mathrm{H} . J=7.5 \mathrm{~Hz}), 1.11$ (s. $3 \mathrm{H}) .1 .12 \cdot 1.16(\mathrm{~m}, 1 \mathrm{H}) .1 .32(\mathrm{~s}, 3 \mathrm{H})$, $1.44-1.50$ (m. IH). 2.26-2.28 (m. lH). 2.29 (s. lH). 3.29 (dd. IH. $J=3.38 .8 .58 \mathrm{~Hz}$ ). 3.51 (dd. $1 \mathrm{H}, J=7.14 .11 .0 \mathrm{~Hz}), 3.63$ (dd, $1 \mathrm{H}, J=4.2,10.9 \mathrm{~Hz}$ ). 3.97-4.01 (nt. 1H) 7.17-7.25 (m, 6H). $7.30-7.37(\mathrm{~m}, 5 \mathrm{H}), 7.66-7.70(\mathrm{~m}, 2 \mathrm{H}):{ }^{i 3} \mathrm{C}$ NMR $(125$ $\mathrm{MHz} . \mathrm{CDCl}_{3}$ ) ô $149.5,149.0 .14+1.2$. 141.1. 140.2, 128.7 , $128.6,128.5,127.8,127.4,126.1$. 126.0, 125.4, 120.2. 120.1. 107.7.80.8. 76.3. 72.9.62.7.53.9.27.0. 26.6.24.3. 10.9. Anal. Calcd for $\mathrm{C}_{2} \mathrm{H}_{31} \mathrm{NO}_{3}$ : C. 77.67 H. 7.48 N. 3.35. Found: C, $77.63:$ H. $7.49 \mathrm{~N}, 3.35$.

General preparation of $13 \mathrm{a}, 13 \mathrm{~b}$ and $13 \mathrm{c}:$ Representative procedure for the preparation of 1-cyclohexyl-1,2-dideoxy3,4-O-isopropylidene-5- $O$-mesy $]-2-[(9-$ phenylf luoren9-yl)amino]- $D$-xylitol (13a). To a solution of 12 a $(0.73 \mathrm{~g} .1 .5 \mathrm{l}$ mmol) in $\mathrm{CH}_{2} \mathrm{Cl}_{2}(30 \mathrm{~mL})$ was added Et $2 \mathrm{~N}(0.32 \mathrm{~mL}, 2.27$ numol) at $0{ }^{\circ} \mathrm{C}$. The reaction nuisture stirred for 20 nin and added $\mathrm{MsCl}(0.15 \mathrm{~mL} .1 .97 \mathrm{mmol})$ and stirred for $30 \mathrm{~min}$. The reaction quenched by addition of $\mathrm{NaHCO}_{3}$ solution $(10 \%, 10$ $\mathrm{mL})$ and the reaction mixture extracted with $\mathrm{CH}_{2} \mathrm{Cl}_{2}(3 \times 30$ $\mathrm{nL}$ ). The combined organic extracts were dried with anhydrous $\mathrm{MgSO}_{4}$ and evaporated under vacuo. The crude mixture was purified by flash column chromatography $\left(\mathrm{CH}_{2} \mathrm{Cl}_{2}\right)$ to afford pure $13 \mathrm{a}(0.84 \mathrm{~g} .99 \%)$ as an oil.

$13 \mathbf{a}[\alpha]_{D}=+11.15\left(c 2.00 . \mathrm{CHCl}_{3}\right): \mathbb{R}(\mathrm{KRS}-5) 3350.2990$. $2920.2850,1740.1450,1360.1250,1250.1180 .750 \mathrm{~cm}^{-1} ;{ }^{l} \mathrm{H}$ NMR $\left(500 \mathrm{MHz}, \mathrm{CDCl}_{3}\right)$ oे $0.22-0.26(\mathrm{~m}, \mathrm{lH}), 0.43-0.47(\mathrm{~m}$. IH). $0.82-0.88(\mathrm{~m} .2 \mathrm{H}) .0 .93-0.98(\mathrm{~m} .3 \mathrm{H}) .0 .99-1.05(\mathrm{~m} . \mathrm{lH})$. $1.13-1.15$ (n. 1H). 1.22-1.27 (m. $5 \mathrm{H}$ ), 1.42-1.49 (n. $5 \mathrm{H}$ ). 1.99 (s. $\mathrm{lH}$ ). 2.22 (s. lH). 3.50 (dd. $\mathrm{IH}, J=2.3 .8 .4 \mathrm{~Hz}$ ). $4.0 \mathrm{l}$ (dd. $1 \mathrm{H} . J=6.3 .11 .1 \mathrm{~Hz}), 4.35$ (dd. $1 \mathrm{H} . J=2.7 .11 .1 \mathrm{~Hz}) .4 .42-$ $4.46(\mathrm{~m}, \mathrm{lH}) .7 .18-7.32(\mathrm{~m}, 8 \mathrm{H}) .7 .37-7.42(\mathrm{~m}, 3 \mathrm{H}) .7 .66-7.7 \mathrm{I}$ $(\mathrm{m}, 2 \mathrm{H}) ;{ }^{13} \mathrm{C} \mathrm{NMR}\left(125 \mathrm{MHz}, \mathrm{CDCl}_{3}\right)$ ô $150.8,149.1,145.0$, $141.1,140.2,128.6,128.4$. 127.9. 127.7, 127.2, 126.2. 125.7. $125.5,120.2,119.9,109.0,78.4,74.6,72.6,69.8,48.3,+1.0$. 37.7. 33.8. 33.6. 32.5. 27.3. 26.8. 26.4. 26.0. 25.9. 14.2. Anal. Calcd for $\mathrm{C}_{32} \mathrm{H}_{41} \mathrm{NO}_{5} \mathrm{~S}: \mathrm{C} .70 .31 \mathrm{H}, 7.33 \mathrm{~N}, 2.48$. Found: $\mathrm{C}$. $70.33 ;$ H. $7.35 \mathrm{~N}, 2.45$.

13b: Yield : $98 \%:[\alpha]_{\mathrm{L}}=+14.27\left(c 2.00 . \mathrm{CHCl}_{3}\right): \mathbb{R}(\mathrm{KRS}-5)$ $3330,3060,2980,2950,2870,1450,1360,1180,960,740$ 
$\left.\mathrm{cm}^{\cdot 1}:{ }^{\mathrm{l}} \mathrm{H} \mathrm{NMR}\left(500 \mathrm{MHz}^{\mathrm{CDCl}}\right)_{3}\right) \delta 0.27(\mathrm{~d}, 3 \mathrm{H}, J=6.5 \mathrm{~Hz})$. 0.47 (d. $3 \mathrm{H}, J=6.5 \mathrm{~Hz}) .0 .74-0.80(\mathrm{~m} . \mathrm{HH}), 1.18-1.33(\mathrm{~m} .3 \mathrm{H})$. $1.30(\mathrm{~s}, 3 \mathrm{H}), 1.50$ (s. $3 \mathrm{H}), 2.02(\mathrm{~s}, 1 \mathrm{H}), 2.15(\mathrm{~s}, 1 \mathrm{H}), 3.01$ (s. $3 \mathrm{H}), 3.55$ (dd, $1 \mathrm{H}, J=2.1 .8 .5 \mathrm{~Hz}) .3 .94$ (dd. $1 \mathrm{H} . J=5.9 .11 .2$ $\mathrm{Hz}), 4.28$ (dd. $1 \mathrm{H}, J=2.8,11.2 \mathrm{~Hz}), 4.46-4.49(\mathrm{~m} .1 \mathrm{H})$. 7.19-7.31 (m, 8H), 7.36-7.42 (m,3H). 7.66-7.71 (m. $2 \mathrm{H}) ;{ }^{3} \mathrm{C}$ NMR (125 MHz, $\left.\mathrm{CDCl}_{3}\right)$ ò 150.8, 149.1, 145.1, 141.0. 140.3. 128.6. 128.4, 127.8, 127.2. 126.1. 125.7, 125.6, 120.1. 119.9. $109.0 .78 .2 .74 .5 .72 .6 .69 .4 .49 .1,42.6 .37 .7 .27 .4 .26 .8 .24 .4$. 23.2.21.3. Anal. Calcd for $\mathrm{C}_{31} \mathrm{H}_{3}: \mathrm{NO}_{5} \mathrm{~S}: \mathrm{C} .68 .81: \mathrm{H} .7 .21: \mathrm{N}$. 7.12. Found: C, 68.83: H. 7.25: N, 7.15

13c: Yield : 95\%: $[\alpha]_{D}=+48.88\left(c\right.$ 2.00. $\left.\mathrm{CHCl}_{3}\right)$. IR (KRS-5) 3340, 2980. 2940, 1730, 1450, 1360, 1250, 1180 , $970.740 \mathrm{~cm}^{-1}:{ }^{1} \mathrm{H} \mathrm{NMR}\left(500 \mathrm{MHz}, \mathrm{CDCl}_{3}\right) \delta 0.54$ (t. $3 \mathrm{H}, J=$ $7.4 \mathrm{~Hz}), 1.14-1.16(\mathrm{~m}, 1 \mathrm{H}) .1 .12-1.18(\mathrm{~m}, 1 \mathrm{H}), 1.30(\mathrm{~s} .3 \mathrm{H})$. 1.47 (s. 3H). $2.03(\mathrm{~m} .1 \mathrm{H}) .2 .21(\mathrm{~s}, 1 \mathrm{H}) .3 .02(\mathrm{~s}, 3 \mathrm{H}) .3 .61$ (dd, IH $J=2.84 .8 .42 \mathrm{~Hz}$ ) $3.96(\mathrm{dd}, 1 \mathrm{H}, J=5.85,11.2 \mathrm{~Hz}), 4.29$ (dd. $1 \mathrm{H}, J=2.6,11.3 \mathrm{~Hz}), 4.37-4.41$ (m, 1H). 7.19-7.27 (m, 7H) $.7 .30-7.33(\mathrm{~m} . \mathrm{lH}) .7 .35-7.38(\mathrm{~m} . \mathrm{IH}) .7 .4 \mathrm{l}-7.42(\mathrm{~m}, 2 \mathrm{H})$. 7.68 (dd. $2 \mathrm{H} . J=7.7 .8 .2 \mathrm{~Hz}) ;{ }^{13} \mathrm{C} \mathrm{NMR}(125 \mathrm{MHz} . \mathrm{CDCl}) \delta$ $151.0 .149 .1 .145 .3,140.6,140.3,128.5,128.4,127.8,127.7$. $127.2,126.1,125.6,125.4,120.1,119.9,109.1 .78 .3,75.0$. $72.5,69.3 .53 .0,37.7,27.3 .26 .8,26.1,14.2 .10 .3$. Anal. Calcd for $\mathrm{C}_{28} \mathrm{H}_{33} \mathrm{NO}_{5} \mathrm{~S}:$ : C. 67.85: H. 6.71: N. 2.83. Found: C. 67.83: $\mathrm{H}, 6.75 ; \mathrm{N}, 2.85$

General preparation of $1+a, 1+b$ and $1+c$ : Representative procedure for the preparation of 1-cyclohexyl-1,2,5-tridideoxy-3,4-0-isopropylidene-5-iodo-2-[(9-phenylfluoren9-yl)amino]- $D$-lyxose (14a). To a solution of 13 a ( $1.2 \mathrm{~g} .2 .13$ mmol) in DMF (20 mL) was added Lil (0.3 $\mathrm{lg}, 2.33 \mathrm{mmol}$ ) at rt. The reaction mixture stirred for $36 \mathrm{~h}$ at $75^{\circ} \mathrm{C}$ and cooled down to it with cold water. To the reaction mixture added saturated $\mathrm{NaHCO}_{3}$ solution ( $10 \mathrm{~mL}$ ) and stirred for $5 \mathrm{~min}$ and extracted with $\mathrm{CH}_{2} \mathrm{Cl}_{2}(3 \times 30 \mathrm{~mL})$. The combined organic extracts were dried with anhydrous $\mathrm{MgSO}_{4}$ and evaporated under vacuo. The cnide mixture was purified by flash column chromatography (hexane/EtOAc, 3:1. $\mathrm{v} / \mathrm{\text { }}$ ) to afford pure $\mathbf{1 4 a}$ $(1.01 \mathrm{~g} .80 \%$ ) as an oil.

14a: $[\alpha]_{\mathrm{D}}=+1+.56\left(c 2.00 . \mathrm{CHCl}_{3}\right)$ : IR (KRS-5) 3330. 3040, 2930. 2850. 1450.980.870,760 $\mathrm{cm}^{-1}$; ${ }^{l} \mathrm{H}$ NMR $(500 \mathrm{MHz}$. $\left.\mathrm{CDCl}_{3}\right)$ oे $1.40-1.66(\mathrm{~m}, \mathrm{loH}), 1.33(\mathrm{~s}, 3 \mathrm{H}) .1 .39$ (s. 3H). $1.42-1.47$ (m. $1 \mathrm{H}$ ). $1.82-1.87$ (m. $2 \mathrm{H}$ ). $2.22-2.43$ (bs. NH), $2.92-3.0 \mathrm{l}(\mathrm{m} . \mathrm{lH}) .3 .23(\mathrm{dd} .2 \mathrm{H}, J=2.8 \mathrm{~Hz}) .3 .3 \mathrm{l}-3.3+(\mathrm{m}$. $1 \mathrm{H}), 3.38-3.41(\mathrm{~m}, 1 \mathrm{H}), 7.16-7.30(\mathrm{~m}, 8 \mathrm{H}), 7.35-7.40(\mathrm{~m}, 3 \mathrm{H})$. $7.63-7.69(\mathrm{~m}, 2 \mathrm{H}):{ }^{13} \mathrm{C} \mathrm{NMR}(125 \mathrm{MHz}, \mathrm{CDCl})$ o 1505,148 7. 144.4. $142.0,141.1,127.7,127.3,126.6,126.3,126.0$. $125.4,124.8 .12+.3 .119 .5 .118 .7,107.4,81.4,74.7,71.4,59.3$. $48.2,44.5,26.6,26.1,22.5,22.3 .22 .1,22.0 .15 .1,6.0$.

14b: Yield : $85 \%:[\alpha]_{D}=+21.34\left(c 2.00 . \mathrm{CHCl}_{3}\right)$ : $\mathbb{R}(\mathrm{KRS}-5)$ $3330,3060,2950,2870,1450,1370,1240,1170,1120,1040$. $890,740 \mathrm{~cm}^{-1}$; ${ }^{1} \mathrm{H} \mathrm{NMR}\left(500 \mathrm{MHz}, \mathrm{CDCl}_{3}\right)$ ồ 0.21 (d. $3 \mathrm{H} . J=$ $6.5 \mathrm{~Hz}), 0.51(\mathrm{~d} .3 \mathrm{H}, J=6.5 \mathrm{~Hz}), 0.67-0.72(\mathrm{~m}, 1 \mathrm{H}), 0.86-0.90$ (m. IH) 1.39 (s. 3H) 1.55 (s. 3H). 2.06 (s. IH) 2.16 (s. IH). 2.88 (dd. $1 \mathrm{H} . J=5.9 .10 .6 \mathrm{~Hz}) .3 .08$ (dd, $1 \mathrm{H} . J=4.5,10.6 \mathrm{~Hz}$ ), 3.49 (dd. $1 \mathrm{H}, J=1.5 .7 .8 \mathrm{~Hz})+.17-4.21(\mathrm{~m} \mathrm{lH}) .7 .18-7.29(\mathrm{~m}$ $8 \mathrm{H}), 7.30-7.31(\mathrm{~m}, 1 \mathrm{H}), 7.36-7.40(\mathrm{~m}, 1 \mathrm{H}), 7.42-7.44(\mathrm{~m}, 2 \mathrm{H})$. 7.66-7.71 (m. 2H): ${ }^{13} \mathrm{C} \mathrm{NMR}\left(125 \mathrm{MHz}, \mathrm{CDCl}_{3}\right) \delta 151.3$. 149.3. 145.4. 141.0.140.3. 128.5, 128.3. 127.9. 127.7. 127.2.
126.2 . 125.8. 125.7. 120.0. 119.9, 108.5, 82.5, 75.5, 72.5, 60.4. $49.4,43.2,27.6,27.4,24.6,23.6,21.0,14.2,6.8$.

$1+\mathrm{c}$ : Yield : $81 \%:[\alpha]_{\mathrm{D}}=+11.56\left(c \cdot 2.00, \mathrm{CHCl}_{3}\right) ; \mathrm{R}(\mathrm{KRS}-5)$ $3430.3060,2980,2930,2870.1450 .1370,1240,1030.730$ $\mathrm{cm}^{-1}:{ }^{\mathrm{l}} \mathrm{H}$ NMR $\left(500 \mathrm{MHz}, \mathrm{CDCl}_{z}\right)$ oे 0.53 (t. $\left.3 \mathrm{H}, J=7.4 \mathrm{~Hz}\right)$. $0.92-0.97(\mathrm{~m}, 1 \mathrm{H}), 1.05-1.11(\mathrm{~m}, 1 \mathrm{H}), 1.39(\mathrm{~s} .3 \mathrm{H}) .1 .51(\mathrm{~s}$, $3 \mathrm{H}) .2 .02$ (s. IH), 2.25 (s. IH) 2.92 (dd. $\mathrm{IH} . J=6.0 .10 .6 \mathrm{~Hz}$ ), $3.12(\mathrm{dd} .1 \mathrm{H} . J=4.0,10.6 \mathrm{~Hz}) .3 .53(\mathrm{dd} .1 \mathrm{H} . J=2.4,7.8 \mathrm{~Hz})$, $4.08-4.11(\mathrm{~m}, \mathrm{lH}), 7.16-7.25(\mathrm{~m}, 5 \mathrm{H}), 7.27-7.30(\mathrm{~m} .2 \mathrm{H})$. $7.30-.32(\mathrm{~m}, \mathrm{lH}), 7.35-7.38(\mathrm{~m}, \mathrm{lH}), 7.42-7.44(\mathrm{~m}, 2 \mathrm{H})$. 7.67-7.70 (m, 2H); ${ }^{13} \mathrm{C}$ NMR (125 MHz, CDCl $) \delta 151.4$, $149.2,145.5,140.5,140.3,128.4,128.3,128.0,127.9,127.7$. 127.1. 126.2, 125.7, 125.4, 120.0. 119.9. 119.6, 108.6. 82.6, $76.0 .72 .4,53.0 .27 .6,27.4 .26 .7,10.2 .6 .9$.

General preparation of $15 \mathrm{a}, 15 \mathrm{~b}$ and $15 \mathrm{c}$ : Representative procedure for the preparation of $(3 S,+5)-4-(9-$ phenyl-9H-fluoren-9-ylamino)-5-cyclohexylpent-1-en-3-ol (15a). To a solution of $14 \mathrm{a}(1.36 \mathrm{~g} .4 .11 \mathrm{mmol})$ in THF $(15 \mathrm{~mL})$ was added $n$-BuLi ( $1.6 \mathrm{M}$ in hexane, $7.69 \mathrm{~mL}, 12.3 \mathrm{mmol}$ ) at $0^{\circ} \mathrm{C}$. The reaction misture stirred for 20 min and quenched by addition of saturated $\mathrm{NH}_{4} \mathrm{Cl}$ solution ( $10 \mathrm{~mL}$ ) and the reaction misture extracted with EtOAc $(3 \times 30 \mathrm{~mL})$. The combined organic extracts were dried with anlyydrous $\mathrm{MgSO}_{4}$ and evaporated under vacuo. The crude mixture was purified by flash column chromatography (hexane/EtOAc. $7: 1$. v/v) to afford pure 15a $(1.57 \mathrm{~g} .90 \%)$ as an oil.

ISa: IR (KRS-5) 3450, 3390, 3310. 3060, 2920, 2850. 1450, $1070.930 .740 \mathrm{~cm}^{-1} ;{ }^{1} \mathrm{H}$ NMR $\left(500 \mathrm{MHz}, \mathrm{CDCl}_{3}\right) \delta 0.27-0.29$ (m, IH). 0.46-0.51 (m, lH). 0.82-0.90 (m, 6H), 1.15-0.31 (m. $2 \mathrm{H}$ ). $1.42-1.50$ (nt. $3 \mathrm{H}$ ), 2.13-2.17 (n. $1 \mathrm{H}), 2.58$ (s, 2H), $3.62-3.64(\mathrm{~m} . \mathrm{lH}), 4.99-5.0 \mathrm{l}(\mathrm{m}, \mathrm{lH}), 5.12-5.16(\mathrm{~m}, \mathrm{lH})$. $5.48-5.55(\mathrm{~m} . \mathrm{lH}), 7.17-7.40(\mathrm{~m} \mathrm{llH}), 7.65-7.71(\mathrm{~m} 2 \mathrm{H}){ }^{12} \mathrm{C}$ $\mathrm{NMR}\left(125 \mathrm{MHz}, \mathrm{CDCl}_{3}\right) \delta$ 150.4, 149.0, 145.3, 141.0, 140.2. $139.6,128.4$. 128.3, 127.8, 127.2. 126.4, 126.1, 125.4. 120.0. $119.9,115.4 .74 .1 .72 .6,54.2,41.6 .34 .0,33.6 .32 .8,26.4$, 26.2. 26.1.14.1

15b: IR (KRS-5) 3570, 3420, 3310, 3060, 2950, 2870, 1450, 1280. 1030. 920.740 $\mathrm{cm}^{-1}:{ }^{1} \mathrm{H}$ NMR (500 MHz. $\left.\mathrm{CDCl}_{3}\right) \delta \mathrm{\delta} 0.31$ $(\mathrm{d}, 3 \mathrm{H} . J=6.2 \mathrm{~Hz}) .0 .51$ (d. $3 \mathrm{H}, J=6.2 \mathrm{~Hz}) 0.80-0.90(\mathrm{~m}, 2 \mathrm{H})$, $1.20-1.36(\mathrm{~m}, 2 \mathrm{H}), 2.09-2.12(\mathrm{~m}, 1 \mathrm{H}), 2.40(\mathrm{~s}, 1 \mathrm{H}), 3.63-3.65$ (m. lH). 4.98 (d. IH. $J=10.5 \mathrm{~Hz}$ ). 5.12 (d. lH. $J=17.2 \mathrm{~Hz}$ ). $5.46-5.51(\mathrm{~m}, 1 \mathrm{H}), 7.19-7.40(\mathrm{~m}, 1 \mathrm{H}), 7.66-7.71(\mathrm{~m} .2 \mathrm{H}){ }^{12} \mathrm{C}$ NMR (125 MHz, $\left.\mathrm{CDCl}_{2}\right)$ ò 148.0. 140.0. 128.4. 127.8, 127.7 . $127.2,126.4,126.0 .125 .5,120.0 .119 .8,115.3,74.0,54.8$, 43.2. $24.5,23.1,21.6$.

15c: IR (KRS-5) $3600,3410,3340.3060 .2960 .2930 .2870$. 1450. 1380. $1280 \mathrm{~cm}^{-1}$ : ${ }^{1} \mathrm{H}$ NMR $\left(500 \mathrm{MHz} . \mathrm{CDCl}_{3}\right)$ ò $0.56-0.59$ (t. $3 \mathrm{H}, J=7.4 \mathrm{~Hz}), 0.84-0.96(\mathrm{~m} .2 \mathrm{H}), 0.99-1.09(\mathrm{~m}$. $1 \mathrm{H}), 1.25(\mathrm{~s}, 1 \mathrm{H}), 2.01-2.12(\mathrm{~m}, 1 \mathrm{H}), 3.71-3.73(\mathrm{~m} \mathrm{lH}), 5.01-5.03$ (m, lH) . 5.14-5.18(m, lH). 5.43-5.50(m. lH). 7.18-7.46(m. $11 \mathrm{H}), 7.68(\mathrm{dd}, 2 \mathrm{H}, J=7.5 .13 .9 \mathrm{~Hz})) ;{ }^{13} \mathrm{C} \mathrm{NMR}(125 \mathrm{MHz}$, $\left.\mathrm{CDCl}_{3}\right)$ ô $143.0,141.5,140.6 .136 .9$. 129.4, $128.6,128.2$. $127.7,126.3,125.8,118.2 .79 .1,67.8,56.3,24.6,9.6$.

General preparation of $16 \mathrm{a}, 16 \mathrm{~b}$ and $16 \mathrm{c}$ : Representative procedure for the preparation of $(3 S, 4 S)-3$-benzyloxy-5-cyclohexyl-4-(9-phenỵl-9H-fluoren-9-ylamino)-1-penten (17a). To a solution of $15 \mathrm{a}(1.20 \mathrm{~g} .3 .54 \mathrm{mmol})$ in THF (15 mL) was added slowly $\mathrm{NaH}(0.15 \mathrm{~g} .6 .37 \mathrm{mmol})$ at $0{ }^{\circ} \mathrm{C}$. The reaction 
mixture stirned for $10 \mathrm{~min}$. and added benzylbromide $(0.72 \mathrm{~g}$. $4.25 \mathrm{mmol}$ ) and stirred for $30 \mathrm{~h}$ at it. The reaction quenched by addition of saturated $\mathrm{NaHCO}_{3}$ solution $(15 \mathrm{~mL})$ and the reaction mixture extracted with EtOAc $(3 \times 30 \mathrm{~mL})$. The combined organic extracts were dried with anhydrous $\mathrm{MgSO}_{4}$ and evaporated under vacuo. The crude mixture was purified by flash column chromatography (hexane/EtOAc, $10: 1, v / v$ ) to afford pure $16 \mathrm{a}(1.6+\mathrm{g} .90 \%)$ as an oil

16a: IR (KRS-5) 3330, 3060, 3030, 2920, 2850, $2360,1450$. 1110. 1070, 740. $700 \mathrm{~cm}^{-1}:{ }^{1} \mathrm{H} \mathrm{NMR}\left(500 \mathrm{MHz} . \mathrm{CDCl}_{3}\right) \delta$ $0.40-0.43(\mathrm{~m}, 1 \mathrm{H}) .0 .54-0.59(\mathrm{~m} .1 \mathrm{H}) .0 .83-0.88$ (m. $2 \mathrm{H})$, $0.99-1.08(\mathrm{~m}, 3 \mathrm{H}), 1.25-1.30(\mathrm{~m}, 3 \mathrm{H}), 1.43-1.45(\mathrm{~m}, \mathrm{lH})$. 1.51-1.53 (m. $2 \mathrm{H}), 1.98$ (5. $\mathrm{HH}), 2.39-2.44$ (m. 1H). 3.28-3.29 (m. IH) 3.90 (d. IH, $J=12.3 \mathrm{~Hz}$ ). 4.04 (d. IH. $J=12.3 \mathrm{~Hz}$ ). 5.15-5.23 (m, 2H). 5.90-5.97 (m. 1H). 7.03-7.07 (m. 2H), $7.13-7.28(\mathrm{~m} .11 \mathrm{H}) .7 .32-7.35(\mathrm{~m} \mathrm{lH}), 7.43-7.45(\mathrm{~m}, 2 \mathrm{H})$. $7.57-7.59(\mathrm{~m} . \mathrm{IH}) .7 .67$ (d. $\mathrm{IH}, J=7.5 \mathrm{~Hz}):{ }^{17} \mathrm{C} \mathrm{NMR}(125$ $\left.\mathrm{MHz} . \mathrm{CDCl}_{7}\right)$ oे 150.4, 150.3, 145.8. 140.8. 139.1. 136.1, 128.4. 128.2. 128.1, 128.0. 127.8, 127.4, 127.2, 127.1, 126.3. 126.2. 125.6, 120.0, 119.7, 81.9. 72.8. 70.1. 52.0. 39.8. 34.0, $33.5,32.9 .26 .6,26.4 .26 .2$

16b: IR (KRS-5) 3330, 3060, 3030,3000, 2870, 1450, 1360. $1110,1070.1030 .740,700 \mathrm{~cm}^{-1} ;{ }^{1} \mathrm{H}$ NMR $\left(500 \mathrm{MHz}, \mathrm{CDCl}_{3}\right)$ ò 0.40 (d. $3 \mathrm{H}, J=6.5 \mathrm{~Hz}) .0 .54$ (d. $3 \mathrm{H}, J=6.5 \mathrm{~Hz}), 0.79-093$ (m, 1H). 1.20-1.30(m, 2H). 1.41-1.48 (m. 1H), 2.38-2.41 (m, IH) $3.30-3.32(\mathrm{~m} . \mathrm{HH}) .3 .91$ (d. IH. $J=12.3 \mathrm{~Hz}) .4 .06(\mathrm{~d} . \mathrm{lH}$. $J=12.3 \mathrm{~Hz}), 5.13-5.22(\mathrm{~m}, 2 \mathrm{H}) .5 .89-5.96(\mathrm{~m} .1 \mathrm{H}), 6.98-7.07$ (m, 2H), 7.19-7.27 (m, 8H), 7.34-7.36 (m, $4 \mathrm{H}), 7.43-7.45(\mathrm{~m}$, $2 \mathrm{H}) .7 .58-7.67(\mathrm{~m} .2 \mathrm{H}):{ }^{1 ?} \mathrm{C} \mathrm{NMR}\left(125 \mathrm{MHz}, \mathrm{CDCl}_{3}\right)$ ò 150.3 . 139.1. 136.1. 128.4, 128.2. 128.1, 128.0, 127.8. 127.7. 127.6. $127.4,127.2,127.1,127.0,126.3,126.1,125.7,119.8,119.7$. $117.1 .82 .1,72.8 .72 .2,70.2,53.0 .41 .4,24.1 .23 .0,22.2$

16c: IR (KRS-5) 3330, 3060, 3030, 2960, 2930, 2870, 1490. 1450. 1230. 1070, $930 \mathrm{~cm}^{-1}:{ }^{1} \mathrm{H}$ NMR $\left(500 \mathrm{MHz}, \mathrm{CDCl}_{3}\right) \hat{o}$ $0.62(\mathrm{t}, 3 \mathrm{H} . J=7.4 \mathrm{~Hz}) .0 .90-0.99(\mathrm{~m}, \mathrm{lH}) .1 .14-1.22(\mathrm{~m} . \mathrm{HH})$, $2.28-2.32(\mathrm{~m} .2 \mathrm{H}) .3 .39-3 .+1(\mathrm{~m} .1 \mathrm{H}) .4 .03$ (d. IH. $J=12.2$ $\mathrm{Hz}), 4.27(\mathrm{~d}, 1 \mathrm{H}, J=12.2 \mathrm{~Hz}), 5.14-5.2+(\mathrm{m} .2 \mathrm{H}), 5.73-5.80$ (m. $\mathrm{lH}), 7.04-7.36(\mathrm{~m}, \mathrm{l}+\mathrm{H}), 7.41-7.46(\mathrm{~m}, 2 \mathrm{H}) .7 .61-7.66(\mathrm{~m}$. $2 \mathrm{H}):{ }^{17} \mathrm{C}$ NMR $\left(125 \mathrm{MHz} . \mathrm{CDCl}_{3}\right) \delta 1505,150.3 .146 .1$. $140.5 .140 .2,138.9,136.0 .128 .4,128.2,128.1 .128 .0,127.8$, $127.7 .127 .5,127.4,127.3,127.2,127.0,126.2,126.0 .125 .8$ $119.7,118.0,82.3 .72 .9,72.2 .70 .2,57.0 .23 .8,10.0$

General preparation of $17 \mathrm{a}, 17 \mathrm{~b}$ and $17 \mathrm{c}:$ Representative procedure for the preparation of $(2 R, 3 R)$-2-benzyloxy-4-cyclohexyl-3-(9-phenyl-9H-fluoren-9-ylamino)-1-pentanoic $\operatorname{acid}(17 \mathrm{a})$. To a solution of $16 \mathrm{a}(1.00 \mathrm{~g} .1 .95 \mathrm{mmol})$ in acetone $(20 \mathrm{~mL})$ was added NMO $(0.66 \mathrm{~g} .6 .23 \mathrm{mmol})$ and catalytic amount of $\mathrm{OsO}_{4}$ at $0^{\circ} \mathrm{C}$. The reaction mixhure stirred for $12 \mathrm{~h}$. and added water $(30 \mathrm{~mL})$ and stirred for $10 \mathrm{~min}$ at $\mathrm{rt}$. The reaction mixture extracted with EtOAc $(3 \times 30 \mathrm{~mL})$. The combined organic extracts were dried with anhydrous $\mathrm{MgSO}_{4}$ and evaporated under vacuo. The crude product dissolved in $\mathrm{EtOH} / \mathrm{H}_{2} \mathrm{O}(\mathrm{I}: \mathrm{l} .20 \mathrm{~mL})$ and added $\mathrm{NaIO}_{4}(0.5+\mathrm{g} .2 .53 \mathrm{mmol})$ at rt. and stirred for $1 \mathrm{~h}$. The solvent was evaporated under vacuo and the crude mixture dissolved in $\mathrm{THF} / \mathrm{H}_{2} \mathrm{O}(1: 1.20 \mathrm{~mL})$ and added $\mathrm{K}_{2} \mathrm{CO}_{3}(0.5+\mathrm{g} .0 .39 \mathrm{mmol})$ and stirred for $5 \mathrm{~h}$. at it. The reaction mixture extracted with EtOAc $(3 \times 30 \mathrm{~mL})$ and combined organic extracts were dried with anhydrous $\mathrm{MgSO}_{4}$ and evaporated under vacuo. The crude mixture was purified by flash column chromatography (hexane/EtOAc, $1: 2 . \mathrm{v} / \mathrm{v}$ ) to afford pure $17 \mathrm{a}(0.78 \mathrm{~g} .75 \%)$ as an oil.

17a: IR (KRS-5) 3380, 3030, 2950.2920 .2840 .1710 .1640 . 1430. $1270.1210,710,670 \mathrm{~cm}^{-1}:{ }^{1} \mathrm{H}$ NMR $\left(500 \mathrm{MHz}, \mathrm{CDCl}_{3}\right)$ ô 1.34-1.36 (m, 2H). 1.40-1.4t (m, 11H). 2.01-2.10 (bs. NH), $3.25-3.34(\mathrm{~m}, \mathrm{lH}) .3 .94$ (d. $\mathrm{lH}, J=12.4 \mathrm{~Hz}) .7 .06-7.07(\mathrm{~m}$. 3H). $7.14-7.19$ (m. $5 \mathrm{H}$ ). $7.34-7.55$ (n. $2 \mathrm{H}$ ). $7.60-7.89$ (n. $8 \mathrm{H}$ ). 11.0 (s. $\mathrm{IH}):{ }^{13} \mathrm{C}$ NMR $\left(125 \mathrm{MHz}, \mathrm{CDCl}_{3}\right)$ o 177.2 . 143.2 . $142.5,141.9,140.3,137.5,137.2,136.4,136.2,129.0,129.2$. $128.9,128.7 .128 .4,128.1,127.9 .127 .6,127.5,127.3 .126 .5$, $126.3 .89 .6,73.9 .67 .0,45.1,35.4 .32 .0 .31 .4,28.1 .27 .5 .25 .1$, 24.6 .

17b: IR (KRS-5) 3390,3060, 2960,2920,2850,1720, 1650. $1450.1270 .1110 .740,700 \mathrm{~cm}^{-1}:{ }^{1} \mathrm{H} \mathrm{NMR}\left(500 \mathrm{MHz} \mathrm{CDCl}_{3}\right)$ ô 0.35 (d, $3 \mathrm{H} . J=6.4 \mathrm{~Hz}) .0 .68$ (d. $3 \mathrm{H}, J=6.4 \mathrm{~Hz}), 0.96-1.01$ (m, lH), 1.24- $1.27(\mathrm{~m}, \mathrm{lH}), 1.33-1.40(\mathrm{~m}, \mathrm{lH}), 1.42-1.48(\mathrm{~m}$. 1H). $2.54-2.59(\mathrm{~m}, 1 \mathrm{H}) .3 .15$ (d. $1 \mathrm{H}, J=4.2 \mathrm{~Hz}), 4.01(\mathrm{~d}, 1 \mathrm{H}, J$ $=12.4 \mathrm{~Hz}), 4.45(\mathrm{~d}, \mathrm{H}, J=12.4 \mathrm{~Hz}) \cdot 6.98-7.05(\mathrm{~m}, 2 \mathrm{H})$. $7.12-7.16(\mathrm{~m}, 1 \mathrm{H}), 7.22-7.37$ (m. $12 \mathrm{H}), 7.44-7.55(\mathrm{~m}, 2 \mathrm{H})$, $7.73-7.76(\mathrm{~m} . \mathrm{IH}):{ }^{13} \mathrm{C}$ NMR $\left(125 \mathrm{MHz}, \mathrm{CDCl}_{3}\right)$ ò 1720 . $147.9,147.0,142.6,141.2,139.7,137.5,129.4,129.2,128.9$. $128.5,128.4 .128 .3,128.0,127.6 .127 .4,125.7,125.6 .124 .8$, $120.5 .120 .3 .72 .5,72.4 .52 .0,39.3 .23 .9,23.2 .21 .6$.

17c: IR (KRS-5) 3330, 3060, 2960. 2930. 2870. 1730. 1600. 1450. $1210.1120 \mathrm{~cm}^{-1}:{ }^{1} \mathrm{H}$ NMR $\left(500 \mathrm{MHz}, \mathrm{CDCl}_{3}\right) \delta 0.70(\mathrm{t}$. $3 \mathrm{H} . J=7.4 \mathrm{~Hz}) .1 .08-1.17(\mathrm{~m}, 1 \mathrm{H}), 1.24-1.27(\mathrm{~m} .1 \mathrm{H})$, $1.63-1.71(\mathrm{~m} .1 \mathrm{H}) \cdot 2.39-2.43(\mathrm{~m}, 1 \mathrm{H}), 3.17(\mathrm{~d}, 1 \mathrm{H} . J=4.3 \mathrm{~Hz})$. $4.00(\mathrm{~d}, \mathrm{lH}, J=12.3 \mathrm{~Hz}), 4.5 \mathrm{l}(\mathrm{d} .1 \mathrm{H}, J=12.3 \mathrm{~Hz}) .7 .0 \mathrm{l}-7.04$ $(\mathrm{m}, 2 \mathrm{H}) .7 .08-7.16(\mathrm{~m}, 1 \mathrm{H}) .7 .21-7.29(\mathrm{~m}, 5 \mathrm{H}), 7.31-7.36(\mathrm{~m}$, 2H). $7.44-7.47(\mathrm{~m}, \mathrm{IH}) .7 .53(\mathrm{~d}, \mathrm{lH}, J=7.5 \mathrm{~Hz}) .7 .72$ (d. IH. $J=7.5 \mathrm{~Hz}){ }^{1{ }^{3} \mathrm{C}} \mathrm{NMR}\left(125 \mathrm{MHz} . \mathrm{CDCl}_{3}\right)$ ô 172.1. 147.6. $147.0,142.7,1+1.3,139.6,137.5,129.4,129.3,129.0,128.4$. $128.3,128.0 .127 .6,127.5,125.7,125.4,124.9,120.5,120.3$. $72.8 .72 .5,55.7 .22 .8,10.5$.

Methy] $N$-[(2S,3S)-2-bemzyloxy-4-cyclohexyl-3-(9-pheny]9H-fluoren-9-ylamino)butanoyl-L-leucinate (18). To a mixed solution of $17(0.57 \mathrm{~g} .1 .08 \mathrm{mmol})$. L-leu-OCH $(0.4 \mathrm{l} \mathrm{g.} 3.24$ mnol) and HOBT in THF (10 mL) with catalytic amount of $p$-TsOH was added dropwise DCC $(0.16 \mathrm{~g}, 1.19 \mathrm{~nm}$ mol $)$ in THF $(+\mathrm{mL})$ at $0^{\circ} \mathrm{C}$. The reaction mixture stirred for $5 \mathrm{~min}$ and added $\mathrm{Et}_{\mathrm{s}} \mathrm{N}(0.22 \mathrm{~mL} .1 .60 \mathrm{mmol})$ and stirred for $5 \mathrm{~h}$ at $\mathrm{rt}$. The reaction mixture filtered with celite sintered glass filter. The organic layer treated with saturated $\mathrm{NaHCO}_{3}$ and extracted with EtOAc $(3 \times 30 \mathrm{~mL})$. The combined organic extracts were dried with anhyydrous $\mathrm{MgSO}_{4}$ and evaporated under vacuo. The crude mixture was purified by flash column cluromatography (hexane/EtOAc. 5:1. v/v) to afford pure $18(0.53 \mathrm{~g} .75$ $\%$ ) as an oil.

IR (KRS-5) 3480,3310,3060, 2960, 2870, 2120, 1740 , 1660. $1450,1210,730 \mathrm{~cm}^{-1} ;{ }^{1} \mathrm{H} \mathrm{NMR}\left(500 \mathrm{MHz}, \mathrm{CDCl}_{2}\right) \delta$ $0.79(\mathrm{t}, 3 \mathrm{H} . J=7.4 \mathrm{~Hz}) .0 .88(\mathrm{~d}, 6 \mathrm{H} . J=6 .+\mathrm{Hz}) .1 .06-1.11$ (n. lH). $1.25-1.29(\mathrm{~m} . \mathrm{lH}) .1 .35-1.38(\mathrm{~m}, \mathrm{lH}) .1 .40-1.49(\mathrm{~m} .2 \mathrm{H})$. 1.57-1.61 (m, 1H) 2.29-2.34 (m. 1H). 2.31-2.32 (m. 1H). 3.25 (d. $\mathrm{IH}, J=9.5 \mathrm{~Hz}$ ) 3.42 (d. $\mathrm{lH}, J=3.5 \mathrm{~Hz}$ ). 3.70 (s. $3 \mathrm{H}$ ) $3.3 \mathrm{l}$ (d. $1 \mathrm{H}, J=11.9 \mathrm{~Hz}) .3 .92($ d. $1 \mathrm{H}, J=11.9 \mathrm{~Hz})+4.51-4.56(\mathrm{~m}$, lH). 6.96-6.99(m. 3H). 7.13-7.18(m.4H). 7.19-7.25 (m. 3H). $7.29-7.33(\mathrm{~m}, 3 \mathrm{H}) .7 .4 \mathrm{l}-7.44(\mathrm{~m} .3 \mathrm{H}) .7 .64-7.67(\mathrm{~m} .2 \mathrm{H}):{ }^{13} \mathrm{C}$ 
$\operatorname{NMR}\left(125 \mathrm{MHz} \mathrm{CDCl}_{3}\right)$ ò $173.0,171.7 .151 .4,150.1 .145 .7$, $140.5,140.2,137.6,128.6,128.4,128.3,128.2,128.1,127.9$. 127.8. 127.5, 126.9. 126.3, 126.2, 119.7, 119.6. 80.2, 72.7, $72.4,56,0.55 .8 .52 .2,50.0 .41,4,35,0.25 .5 .24 .9,24.8 .24 .7$. $24.2,22.8,22.0,11.4$

$N$-[(2S,3S)-3-Amino-2-hy droxy-4-cyclohexylbutanoy]]L-leucine (20). To a solution of $18(0.80 \mathrm{~g} .1 .22 \mathrm{mmol})$ in $\mathrm{THF} / \mathrm{H}_{2} \mathrm{O}(2: 1,20 \mathrm{~mL})$ was $\mathrm{LiOH}(0.05 \mathrm{mg} .2 .4 \mathrm{mmol})$ at $0^{\circ} \mathrm{C}$. The reaction mixture stirred for $3 \mathrm{~h}$ at $\mathrm{rt}$. and added $3 \% \mathrm{HCl}$ ( 15 $\mathrm{mL})$. The reaction mixture extracted with $i-\mathrm{PrOH} / \mathrm{CH}_{2} \mathrm{Cl}_{2}(\mathrm{l}: 3$. $40 \mathrm{~mL}$ ) and the combined organic extracts were dried with anlydrous $\mathrm{MgSO}_{4}$ and evaporated under vacuo to give cnide product 19. The crude product 19 was hydrogenated with $10 \%$ $\mathrm{Pd} / \mathrm{C}(0.09 \mathrm{~g})$ in $\mathrm{MeOH}(15 \mathrm{~mL})$ at $70^{\circ} \mathrm{C}$ for $10 \mathrm{~h}$. The reaction mixture was filtered through celite and evaporated under vacuo to give crude solid product. The filterate and solid were subjected to ion-exchange chromatography (Dowex 50W-X8. eluting $3 \mathrm{~N} \mathrm{NH}_{3}$ in $\left.\mathrm{H}_{2} \mathrm{O}\right)$ to afford pure $20(0.32 \mathrm{~g} .83 \%$ ) as a solid. mp 201-203 ${ }^{\circ} \mathrm{C}:[\alpha]_{D}=-13.6(\mathrm{C} 1.50,1 \mathrm{~N} \mathrm{HCl}$ ): IR (KRS-5) 3400, 3360. 3350, 2970, 2820, 1720. 1670, 1470, $1200.670 \mathrm{~cm}^{. j}:{ }^{l} \mathrm{H}$ NMR $\left(500 \mathrm{MHz}, \mathrm{D}_{2} \mathrm{O}\right) \delta 0.90($ d. $6 \mathrm{H} . J=$ $6.4 \mathrm{~Hz}), 1.25-1.47(\mathrm{~m}, 13 \mathrm{H}), 1.74(\mathrm{~m}, 2 \mathrm{H}), 1.85(\mathrm{~m} .1 \mathrm{H}), 2.90$ $(\mathrm{m}, 1 \mathrm{H}), 4.39(\mathrm{~d} .1 \mathrm{H}, J=8.9 \mathrm{~Hz}), 4.42(\mathrm{~d} .1 \mathrm{H}, J=13.5 \mathrm{~Hz}){ }^{13} \mathrm{C}$ NMR $\left(125 \mathrm{MHz}, \mathrm{CDCl}_{3}\right) \delta$ 174.9. 172.7. 73.1. 53.5. 52.4 . 41.2 , 35.9. 33.5. 33.2. 31.4. 29.6. 28.9. 25.8. 23.6. 23.3. 22.1 . Anal. Calcd for $\mathrm{C}_{16} \mathrm{H}_{31} \mathrm{~N}_{2} \mathrm{O}_{4}:$ C. 61.12: H. 9.62: N. 8.91. Found: C. 61.15: H, 9.65; N. 8.89.

\section{References and Notes}

1. (a) Cole, D. C. Tetrahedron 1994, 50, 9517. (b) Juaristi, E.; Quintanta. D.: Escalante. I. Aldirinimica Acta 1994. 27, 3. (c) Mclnemey, B. V: Tavlor, W. C. Strdies in Kantrol Products Chemistm: Atta-l1-Rahman, Ed.; Elsevier Science: Amsterdam, 1995: vol. 15, p 381 and references cited therein. (d) Hill, R. A. Prog. Chem. Org. Nat. Prod. 1986, 49, 1. (e) Enantioselective Sinthesis of B-Anmo Acids; Eusebio Tuarsti, Ed.; Wiley-VCH: New York, 1997

2. (a) Rich, D. H. J. Med. (hem 1985, 28, 263. (b) Maibaum, J.: Rich, D. H. J. Org. Chem. 1988, 53, 689. (c) Morishma, H.: Umezawa, H. J. Antibiotics 1973, 26, 115.

3. (a) Lizuka, K.: Kami.To, T.; Harada, H.: Akhane, K.; Kubota, T.;
Umerama, H: Kiso, Y.J. Chem. Soc. Chem. Conmun. 1989, 1678.

4. (a) Kobayashi, Y:; Tákemoto, Y.: Kami,To, T.: [to, Y.: Terashima, S. Tetrahedron 1992, 18, 1853 . (b) OTima, L.; Park. Y. H.; Sun. S. M.: Brigalld, T.; Zhao, M. Tetrahedron Lett. 1992,33,5737. (c) Hattoro, K: Yamamoto, H. Tetrahedron 1994, 50, 2785. (d) Kobayashi, Y.: Takemoto, Y: Kami.To, T.: Ito, Y.: Terashima, S. Tenahedron 1990, 31, 3031. (e) Matsumoto, T:; Kobayashi, Y.; Takemoto, Y: Ito, Y: Kamito, T: Harada, H: Terashima, S. Tetrahedron Left. 1990, 48, 1853. (f) Inokuchi. T.: Tanigawa, S.: Karazaki, M; Torii, S. SinLett 1991, 701. (g) Righi, G.; Rumboldt, G. J. Org. Chent. 1996, 61, 3557. (h) Pasto, M:; CasteJon, P.; Moyano, A.; Hericas, M A.; Riera, A. J. Org. Chent. $1996,61,6033$

5. Lee, J. H.; Lee, B. W.; Tang, K. C.; Teong, I. Y.; Yang, M. S.; Lee, S. G.; Park, K. H. Symthesis 2003, 829.

6. (a) Park, K. H.: Yoon, Y. T.: Lee, S. G. J. Chem Soc Perkin Trans. I 1994, 2621. (b) Csuk, R.; Hugemer, M.: Vasella, A. Heh? Chim. Acta 1998, 71, 609 (c) Zhou, P.: Salleh, H.: Chan, P. C.: LaJioe, G.; Honex, I. F.: Nambiar, P. T. C.: Ward, O. P. Carbohnd Res. $1993,239,155$

7. (a) Chong. I. M.: Cry, D. R.: Mar, E. K. Tetrahedron Lett. 1987. 28, 5009. (b) Alexakis, A.: Tachiet, D.: Nomant, T. F. Tetrahedron 1986, 42, 5607. (c) Berkowitz, D. B.; Chisowa, E.; Mcr adden, . . M. Tetrahedron 2001, 57,6329.

8. Green, T. W. Wuts, P. G. M. Protective in Organic Synthesis, $2^{\text {nut }}$ ed.; Joln Wiley \&Sons, Inc.: $1991 ; p 67$.

9. (a) Park, K. H. Yoon, Y. T.; Lee, S. G. Tetwhed don Lett. 1994, 35. 9737. (b) Lee, T. H.; Lee, B. W.; Jang, K. C., Teong, I. Y; Yang, M. S.; Lee, S. G.; Park, K. H. Sinthesis 2003,829 . (c) Park, S. H.; Lee, H. W. Bull. Korean Chent Sac 2008, 29, 1447

10. (a) Xu, Z; Johannes, C. W.; Houri, A. F, La, D. S.; Cogan, D. A: Hofilena, G. E.; Hoveyda, A. H. J. Am. Chem. Soc. 1997, 119, 10302. (b) Yates, M. H. Tetrahedron Letr. 1997, 38, 2813. (c) Branco, L. C.: Atonso, C. A. M. J. Org. Chem. 2004, 69, 4318. (d) Choudary, B. M.: Chodari, N. S.: Jyothi, K.; Kantam, M. L. J. Am. Chem. Soc. 2002, 124, 5341, (e) Molander, G. A.: Figuleroa, R. Org. Lett. 2006, 8, 75 .

11. Tobe, H.: Morishima, H.: Takita, T.: Aoyagi, T.: Umezawa, H. Agr. Biol Chem. 1979, 43,591.

12. Aoyagi, T.; Tobe, H.; Ko.Tna, F.; Hamada, M.; Takeuchi, T:; Umesawa, H. I. Antiobiot 1978, 31, 221. (b) Puirous. T.; Loughton, B. G. Awh. of Insect Biochen. Phy 1992, 19, 193

13. (a) Regeling, $\mathrm{H}$; de Rouville, E.; Chittenden, G. J. F. Rect Tron: Chim. PavsBas 1987, 106, 461. (b) Csuk, R: Hugemer, M: Vasella, A. Helv Chim . Act. 1988, 71, 609. (c) Lee, S. G. Teong, H. T. Bull. Korean Chem. Sac $1998,19,598$. (d) Lee, S. G.; Lee, J. H. Bull. Korean Chem. Soc. 1997, 18, 556 